\title{
Explicit filtering to obtain grid-spacing-independent and discretization-order-independent large-eddy simulation of compressible single-phase flow
}

\author{
Senthilkumaran Radhakrishnan ${ }^{1}$ and Josette Bellan ${ }^{1,2} \dagger$ \\ 1 Jet Propulsion Laboratory, California Institute of Technology, Pasadena, CA 91109, USA \\ ${ }^{2}$ California Institute of Technology, Pasadena, CA 91125, USA
}

(Received 10 March 2011; revised 5 December 2011; accepted 3 February 2012;

first published online 6 March 2012)

In large-eddy simulation (LES), it is often assumed that the filter width is equal to the grid spacing. Predictions from such LES are grid-spacing dependent since any subgridscale (SGS) model used in the LES equations is dependent on the resolved flow field which itself varies with grid spacing. Moreover, numerical errors affect the flow field, especially the smallest resolved scales. Thus, predictions using this approach are affected by both modelling and numerical choices. However, grid-spacing-independent LES predictions unaffected by numerical choices are necessary to validate LES models through comparison with a trusted template. First, such a template is created here through direct numerical simulation (DNS). Then, simulations are conducted using the conventional LES equations and also LES equations which are here reformulated so that the small-scale-producing nonlinear terms in these equations are explicitly filtered (EF) to remove scales smaller than a fixed filter width; this formulation is called EFLES. First, LES is conducted with four SGS models, then EFLES is performed with two of the SGS models used in LES; the results from all these simulations are compared to those from DNS and from the filtered DNS (FDNS). The conventional LES solution is both grid-spacing and spatial discretization-order dependent, thus showing that both of these numerical aspects affect the flow prediction. The solution from the EFLES equations is grid independent for a high-order spatial discretization on all meshes tested. However, low-order discretizations require a finer mesh to reach grid independence. With an eighth-order discretization, a filter-width to grid-spacing ratio of two is sufficient to reach grid independence, while a filter-width to grid-spacing ratio of four is needed to reach grid independence when a fourth- or a sixth-order discretization is employed. On a grid fine enough to be utilized in a DNS, the EFLES solution exhibits grid independence and does not converge to the DNS solution. The velocity-fluctuation spectra of EFLES follow those of FDNS independent of the grid spacing used, in concert with the original concept of LES. The reasons for the different predictions of conventional LES or EFLES according to the SGS model used, and the different characteristics of the EFLES predictions compared to those from conventional LES are analysed.

Key words: compressible turbulence, turbulence modelling, turbulence simulation 


\section{Introduction}

For several decades, large-eddy simulation (LES) has been considered the most promising methodology for computing fully turbulent flows. Recently LES has been applied to study many industrially relevant flows, though many issues related to the effect of modelling and numerical choices on the LES prediction are still poorly understood. The LES equations are obtained by filtering the governing equations at a filter scale in the inertial range that removes information from the filter scale through the dissipation range. Thus, the resolved field from the LES equations contains the same information at the larger-than-filter scales as the original governing equations but the effects of the unresolved, small-scale (called subgrid scales (SGS)) field appear as additional SGS terms in the LES equations. The SGS terms, being dependent on the unresolved small-scale field, cannot be computed directly from the LES solution, and thus must be modelled as a function of the only known entity, the resolved field of the LES solution. In the most advanced SGS models, each SGS model is the product of a coefficient, computed dynamically as the simulation proceeds along (Germano et al. 1991), multiplied by a functional form of LES variables. Thus, the contribution from the coefficient and functional form to each of the SGS terms in the governing equations is dependent on the resolution at which the LES solution was obtained.

There is a vast literature devoted to examining the optimal filter-width to gridspacing ratio that minimizes numerical error (e.g. Ghosal 1996; Chow \& Moin 2003). Because the SGS contribution in the LES equations is intertwined with the LES grid spacing, the solution can no longer be expected to asymptotically converge with grid refinement. However, the LES formulation is far from being entirely established and alternative formulations have recently been proposed by Fox (2003), and by Pope (2010) enlarging that proposed by Fox (2003).

Until recently, and to be elaborated further below, it was an accepted fact that LES is grid-spacing dependent (Pope 2004). Any prediction from LES is affected both by the modelling and numerical choices. Finite-difference schemes introduce numerical errors because of their inability to accurately calculate the derivatives in the highest resolved wavenumber region or the smallest resolved scales. The intertwining of numerical and modelling errors was highlighted by Vreman, Geurts \& Kuerten (1996) who separated numerical errors from modelling errors for the total kinetic energy in the context of a temporal mixing layer and concluded that the total error is a complex function of the two errors, which sometimes add and other times partially cancel each other. This coupling between errors is not conducive to experimental validation of a model since it is impossible to isolate and eliminate the numerical error, and only highlight the modelling error. On the other hand, separating numerical and modelling errors as was done in the enlightening study of Vreman et al. (1996) may not be feasible under practical conditions. The only acceptable procedure for applications is to eliminate numerical errors and evaluate the model which is then uncontaminated by numerical choices in discretization order and grid spacing. In these applications, only the filter size can be specified (i.e. the user decision that only information above a certain scale is of interest) while the necessary grid spacing is generally unknown.

Realizing the grid-spacing dependence in LES, Geurts \& Frohlich (2002), Meyers, Geurts \& Baelmans (2003, 2005) and Meyers, Sagaut \& Geurts (2006) addressed grid optimization for the purpose of obtaining an LES solution within a specified acceptable error compared to a trusted template; the total error was considered to stem from both modelling and discretization errors. The acceptable error was specified on one or several user-selected objectives, and the selection of the grid involved 
performing a large number of LES realizations to find the path of optimal refinement for a single objective or a path of optimal (or nearly optimal) refinement trajectory in the plane of a variable proportional to the ratio of the filter to grid widths and the inverse of the grid spacing for multiple objectives. Those studies were performed for isotropic and homogeneous turbulence (Meyers et al. 2003, 2005, 2006) for which objectives were each represented by a global quantity, or for a mixing layer (Geurts \& Frohlich 2002) for which the objective was an integral quantity. The large number of necessary LES realizations to be performed, the fact that it is difficult to generalize the procedure for spatially-dependent and time-dependent objectives and, most important the absence of universality in the ensemble of all situations, makes this method hard to implement for complex flows.

The study of Carati, Winckelmans \& Jeanmart (2001) lucidly addressed the sources of information loss in LES and identified the grid to play a pivotal role in LES as it is the reason that information is lost in LES and must be reintroduced through SGS models. Filtering also removes information, but, as an example, for an invertible filter that information can be reconstructed to a required extent from the LES solution; however, the reconstruction can necessarily only be performed on the LES grid. Such reconstruction techniques have been already proposed in the literature (Geurts 1997; Stolz \& Adams 1999; Stolz, Adams \& Kleiser 2001). It has been argued both that the SGS model is physical and that it is not so (Pope 2004). In the latter argument it has been asserted that the SGS model only serves to introduce the correct amount of dissipation in LES. Also to be questioned is the relationship between the LES grid spacing, the filter width and the smallest scales produced in an LES solution.

Applying explicit filtering in order to remove the scales of the motion contaminated by numerical errors has the potential to lead to solutions which are only affected by modelling error. Explicit filtering is not a new concept, as it has been proposed in the past (e.g. Winckelmans et al. 2001; Gullbrand \& Chow 2003; Lund 2003; Mathew et al. 2003; Bose, Moin \& You 2010), but only in some studies was the explicit goal that of obtaining grid-independent solutions (e.g. Bose et al. 2010). For incompressible turbulent isotropic decay, Winckelmans et al. (2001) found that despite favourable $a$ priori assessments, the LES results obtained with or without explicit filtering were similar when using the Smagorinsky model, and that the mixed model only displayed slight improvements when using explicit filtering. Gullbrand \& Chow (2003), Lund (2003) and Bose et al. (2010) all focused on incompressible channel flow. Lund (2003) found that explicit filtering can be used to control the impact of numerical errors and also compared it with the strategy of mesh refinement to increase the accuracy of simulations; Gullbrand \& Chow (2003) highlighted the increased accuracy in the prediction of turbulence intensities when using explicit filtering in conjunction with reconstruction of the resolved subfilter scales (the SGS scales); while Bose et al. (2010) showed that it is possible to obtain grid-independent LES solutions by explicitly filtering the conventional LES equations. This independence can be achieved in LES by keeping the filter width constant and by refining the grid successively; indeed, Magnient, Sagaut \& Deville (2001) recognized that when evaluating the influence of grid resolution, the filter width should be fixed. If for a specified discretization scheme one obtains a solution that no longer depends on grid spacing, then the only source of the error in the grid-spacing-independent solution is due to the SGS model. Explicit filtering could also have advantages in situations where the grid spacing varies over the extent of the domain. Vanella, Piomelli \& Balaras (2008) show that when the grid is suddenly coarsened, considerable energy accumulates at small scales near the interface between the coarse grid and the fine 
grid and it was found that by explicit filtering the convective term, the LES solution is improved because the scales that cannot be represented on the coarse mesh are damped. When explicit filtering is not part of the model equations but only applied to the solution at selected, or all, time steps (Mathew et al. 2003), then the solution is not necessarily grid-spacing independent, and in fact it may improve with grid refinement as shown for compressible channel flow (Mathew et al. 2003).

In the present study we inquire whether by reformulating the LES equations using explicit filtering one can obtain, for compressible flows, LES solutions which are independent of: (i) grid spacing for a given filter width, and (ii) order of spatial discretization. The compressible flows of interest are those associated with internal combustion chambers where the Mach number is in the low subsonic regime. We also wish to investigate how close the solution of the reformulated LES is to a trusted template. Because our investigation is in the context of temporal shear layers for single-phase flows, so as to remove the effect of complex boundary conditions, experimental data are unobtainable and thus the present template is a DNS database. In $\S 2$ we briefly recall the fundamental governing equations of Okong'o \& Bellan (2004). The LES equations are presented in $\S 3$ both for conventional LES and an explicitly-filtered LES, labelled EFLES. In conventional LES, grid dependence is due to the fact that as one refines the grid, a larger portion of turbulent kinetic energy is resolved, whereas in EFLES, when refining the grid, we remove scales smaller than the filter width. We achieve this removal by explicitly filtering these scales at the LES-equations level. To facilitate a comparison between the conventional and explicitly-filtered LES formulations, models of the governing equations, SGS models and coefficient computation for these LES models are presented in parallel in $\S \S 3.1-3.3$. The initial and boundary conditions are described in $\S 4$ while the numerical method, which is different from that of Okong'o \& Bellan (2004), is presented in $\S 5$. In $\S 6$ we first succinctly describe the DNS database in $\S 6.1$, then we list quantities through which we evaluate the LES and EFLES performance in $\S 6.3$, and we compare the conventional LES and EFLES solutions to their chosen template in $\S \S 6.4$ and 6.5 , respectively. Finally, in $\S 6.6$, we explain the results by scrutinizing the SGS dissipation. A summary, conclusions and a discussion of future work are offered in $\S 7$.

\section{Governing equations for direct numerical simulation}

As in Okong'o \& Bellan (2004), we define the vector of gas-phase conservative variables $\phi=\left\{\rho, \rho u_{i}, \rho e_{t}\right\}$ and denote the flow field as $\phi$, where $\rho$ is the density, $u_{i}$ is the velocity in the $x_{i}$ coordinate direction and $e_{t}$ is the total energy. The conservation equations are:

$$
\begin{gathered}
\frac{\partial \rho}{\partial t}+\frac{\partial\left(\rho u_{j}\right)}{\partial x_{j}}=0 \\
\frac{\partial\left(\rho u_{i}\right)}{\partial t}+\frac{\partial\left(\rho u_{i} u_{j}\right)}{\partial x_{j}}=-\frac{\partial p}{\partial x_{i}}+\frac{\partial \sigma_{i j}}{\partial x_{j}}, \\
\frac{\partial\left(\rho e_{t}\right)}{\partial t}+\frac{\partial\left(\rho e_{t} u_{j}\right)}{\partial x_{j}}=-\frac{\partial\left(p u_{j}\right)}{\partial x_{j}}-\frac{\partial q_{j}}{\partial x_{j}}+\frac{\partial\left(\sigma_{i j} u_{i}\right)}{\partial x_{j}},
\end{gathered}
$$

where the thermodynamic variables to be computed from $\phi$ are the internal energy $\left(e=e_{t}-e_{k}\right.$, where the kinetic energy is $\left.e_{k}=u_{i} u_{i} / 2\right)$, the pressure $(p)$, the temperature $(T)$ and the enthalpy $(h=e+p / \rho)$. We assume that the perfect gas 
equation of state

$$
p(\phi)=\rho R(\phi) T(\phi),
$$

holds where $R(\phi)=R_{u} / m_{C}, R_{u}$ is the universal gas constant and $m_{C}$ is the molar mass of the gas and

$$
h(\phi)=C_{p}(\phi) T(\phi),
$$

where the heat capacity at constant $p$ is $C_{p}(\phi)$. To compute $T$, we use the internal energy

$$
e(\phi)=C_{v}(\phi) T(\phi)
$$

where $C_{v}$ is the heat capacity at constant temperature $\left(C_{v}=C_{p}-R\right)$.

In (2.1)-(2.3), $\sigma_{i j}$ is the viscous stress, and $S_{i j}$ is the rate of strain

$$
\begin{gathered}
\sigma_{i j}(\phi)=2 \mu\left(S_{i j}-\frac{1}{3} S_{k k} \delta_{i j}\right), \\
S_{i j}(\phi)=\frac{1}{2}\left(\frac{\partial u_{i}}{\partial x_{j}}+\frac{\partial u_{j}}{\partial x_{i}}\right),
\end{gathered}
$$

where $\mu$ is the viscosity. The heat flux is

$$
q_{j}(\phi)=-\lambda \frac{\partial T(\phi)}{\partial x_{j}},
$$

where $\lambda$ is the thermal conductivity. In (2.7) and (2.9), $\mu$ and $\lambda$ are assumed constant, and are related through the Prandtl number, $\operatorname{Pr}=\mu C_{p} / \lambda$.

\section{Large-eddy-simulation formulations}

In LES, the large-scale part of the flow field is obtained by applying a filtering operation to the governing equations. The filtering operation is defined as

$$
\bar{\psi}(\boldsymbol{x})=\int_{V_{f}} \psi(\boldsymbol{y}) G(\boldsymbol{x}-\boldsymbol{y}) \mathrm{d} \boldsymbol{y}
$$

where $G$ is the filter function and $V_{f}$ is the filtering volume; $G$ has the property that for a spatially invariant function, the filtered function is identical to the unfiltered one. For compressible flows, Favre filtering is used, which is defined as $\tilde{\psi}=\overline{\rho \psi} / \bar{\rho}$.

We adopt the same equation of state and transport properties employed in DNS, and thus in the following, we discuss only the formulation of the governing equations.

\subsection{Governing equations}

\subsubsection{Conventional LES governing equations}

The filtered gas-phase equations are

$$
\begin{gathered}
\frac{\partial \bar{\rho}}{\partial t}+\frac{\partial\left(\bar{\rho} \tilde{u}_{j}\right)}{\partial x_{j}}=0 \\
\frac{\partial\left(\bar{\rho} \tilde{u}_{i}\right)}{\partial t}+\frac{\partial\left(\bar{\rho} \tilde{u}_{i} \tilde{u}_{j}\right)}{\partial x_{j}}=-\frac{\partial[p(\bar{\phi})]}{\partial x_{i}}+\frac{\partial \sigma_{i j}(\bar{\phi})}{\partial x_{j}}-\frac{\partial \tau_{i j}}{\partial x_{j}}
\end{gathered}
$$




$$
\begin{aligned}
\frac{\partial\left(\bar{\rho} \tilde{e}_{t}\right)}{\partial t}+\frac{\partial\left(\bar{\rho} \tilde{e}_{t} \tilde{u}_{j}\right)}{\partial x_{j}}= & -\frac{\partial\left[p(\bar{\phi}) \tilde{u}_{j}\right]}{\partial x_{j}}-\frac{\partial q_{j}(\bar{\phi})}{\partial x_{j}}+\frac{\partial\left[\sigma_{i j}(\bar{\phi}) \tilde{u}_{i}\right]}{\partial x_{j}} \\
& -\frac{\partial \zeta_{j}}{\partial x_{j}}-\frac{\partial}{\partial x_{j}}\left(\tau_{i j} \tilde{u}_{i}\right) .
\end{aligned}
$$

In the above equations (3.2)-(3.4), it is assumed, based on the a priori study of Okong'o \& Bellan (2004), that $\overline{f(\phi)}$ can be replaced by $f(\bar{\phi})$ for pressure, viscous stresses and heat conduction terms, as it was found that the error due to this assumption is negligible. Quantities $\tau_{i j}$ and $\zeta_{j}$ in (3.2)-(3.4) are

$$
\tau_{i j}=\overline{\rho u_{i} u_{j}}-\bar{\rho} \tilde{u}_{i} \tilde{u}_{j}, \quad \zeta_{j}=\overline{\rho h u_{j}}-\bar{\rho} \tilde{h} \tilde{u}_{j},
$$

being the SGS terms that represent the effect of the unresolved part of the flow field on the resolved part of the flow field, where $\tilde{h}=\tilde{e}+\bar{p} / \bar{\rho}$.

In the conventional approach, the equation of state is

$$
p(\bar{\phi})=\bar{\rho} R(\bar{\phi}) T(\bar{\phi}) .
$$

\subsubsection{Explicitly filtered LES (EFLES) governing equations}

In the conventional LES formulation, the filter shape is not specified and is unknown; the only effect of filtering is through the relationship between filter width and grid spacing, making the filter implicit. The truncation error may also act as an implicit filter. Clearly, this lack of knowledge regarding the filter shape poses a problem when comparing LES numerical predictions with experiments since it is uncertain how the raw experimental data should be treated for comparison with simulations. To remedy this situation, one can reformulate the LES equations by introducing an explicit filter to impose a baseline for comparing experiments and simulations. In simulations, this explicit filter has the role of suppressing higher frequencies that arise due to nonlinearities, thereby controlling the spectral content of the resolved flow field. Thus, in explicitly filtered LES, non-linear of the convective terms in the mass, momentum and energy equations, the term describing pressureresponsible work in the energy equation, as well as in the equation of the state are explicitly filtered out; an exception to explicit filtering of all nonlinear terms in the governing equations is the resolved-stress work term in the energy equation, the ad hoc hypothesis being here (justified by the results in $\S 6.5$ ) that this term does not produce significant scales smaller than the spatial grid. Considering that in compressible flows, dynamics and thermodynamics are locally coupled, it is deemed important to filter the convective term in the continuity equation. Applying an explicit filter to the aforementioned nonlinear terms yields

$$
\begin{gathered}
\frac{\partial \bar{\rho}}{\partial t}+\frac{\partial\left(\overline{\bar{\rho} \tilde{u}_{j}}\right)}{\partial x_{j}}=0, \\
\frac{\partial\left(\bar{\rho} \tilde{u}_{i}\right)}{\partial t}+\frac{\partial \overline{\left(\bar{\rho} \tilde{u}_{i} \tilde{u}_{j}\right)}}{\partial x_{j}}=-\frac{\partial[p(\bar{\phi})]}{\partial x_{i}}+\frac{\partial \sigma_{i j}(\bar{\phi})}{\partial x_{j}}-\frac{\partial \tau_{i j}^{e f}}{\partial x_{j}}, \\
\frac{\partial\left(\bar{\rho} \tilde{e}_{t}\right)}{\partial t}+\frac{\partial \overline{\left(\bar{\rho} \tilde{e}_{t} \tilde{u}_{j}\right)}}{\partial x_{j}}=-\frac{\partial \overline{\left[p(\bar{\phi}) \tilde{u}_{j}\right]}}{\partial x_{j}}-\frac{\partial q_{j}(\bar{\phi})}{\partial x_{j}}+\frac{\partial\left[\sigma_{i j}(\bar{\phi}) \tilde{u}_{i}\right]}{\partial x_{j}} \\
-\frac{\partial \zeta_{j}^{e f}}{\partial x_{j}}-\frac{\partial}{\partial x_{j}}\left(\tau_{i j}^{e f} \tilde{u}_{i}\right),
\end{gathered}
$$


and the equation of state is

$$
p(\bar{\phi})=\overline{\bar{\rho} \tilde{R}(\bar{\phi}) \tilde{T}(\bar{\phi})},
$$

where quantities $\tau_{i j}^{e f}$ and $\zeta_{j}^{e f}$ in (3.8)-(3.9) are the SGS terms for the explicitly filtered formulation (denoted by superscript $e f$ )

$$
\tau_{i j}^{e f}=\overline{\rho u_{i} u_{j}}-\overline{\bar{\rho} \tilde{u}_{i} \tilde{u}_{j}}, \quad \zeta_{j}^{e f}=\overline{\rho h u_{j}}-\overline{\bar{\rho} \tilde{h} \tilde{u}_{j}} .
$$

The convective term in the continuity equation is filtered so as to be consistent with filtering of convective terms in the other equations. The term $\partial\left(\bar{\rho} \widetilde{u}_{i}-\overline{\bar{\rho}} \widetilde{u}_{i}\right) / \partial x_{i}$ is considered negligible in the continuity equation as the primary goal of the present study is grid-spacing and discretization-order independence, with accuracy being a secondary goal; this term could be modelled to enhance the LES accuracy if this is deemed necessary in an application. Both $\tau_{i j}^{e f}$ and $\zeta_{j}^{e f}$ are different from the equivalent SGS terms appearing in (3.2)-(3.4).

\subsection{SGS models}

\subsubsection{Conventional LES SGS models}

Four types of models are employed for the SGS terms $\left(\tau_{i j}, \zeta_{j}\right)$, as follows.

(a) The Smagorinsky (Smagorinksy 1963, 1993) model is an eddy viscosity model derived assuming that the production of the SGS turbulent kinetic energy is in balance with the dissipation of the SGS turbulent kinetic energy. In this model

$$
\tau_{i j}-\frac{1}{3} \tau_{k k} \delta_{i j}=-2 C_{S M}^{2} \bar{\Delta}^{2} \bar{\rho}|S(\bar{\phi})|\left[S_{i j}(\bar{\phi})-\frac{\delta_{i j}}{3} S_{k k}(\bar{\phi})\right],
$$

where $S^{2}(\phi)=2 S_{i j}(\phi) S_{i j}(\phi)$ and the Yoshizawa (1986) (YO) model is used to compute

$$
\tau_{k k}=C_{Y O} \bar{\Delta}^{2} S^{2}(\bar{\phi}) .
$$

The SGS scalar enthalpy flux is modelled as

$$
\zeta_{j}=-C_{S M}^{2} \frac{\bar{\Delta}^{2} \bar{\rho}|S(\bar{\phi})|}{P r_{s g s}} \frac{\partial \tilde{h}}{\partial x_{j}} .
$$

Coefficients $C_{S M}$ and $P r_{s g s}$, which is interpreted as an SGS Prandtl number, are computed through the dynamic coefficient computation described in $\S 3.3 .1$ and the model name is abbreviated as LES-DSM.

(b) The gradient model is derived using a Taylor series expansion for the filtered term in the SGS terms (Clark, Ferziger \& Reynolds 1979; Liu, Meneveau \& Katz 1994; Okong'o \& Bellan 2004) to give

$$
\begin{gathered}
\tau_{i j}=-C_{G R S} \bar{\Delta}^{2} \bar{\rho} \frac{\partial \tilde{u}_{i}}{\partial x_{k}} \frac{\partial \tilde{u}_{j}}{\partial x_{k}}, \\
\zeta_{j}=-C_{G R H} \bar{\Delta}^{2} \bar{\rho} \frac{\partial \tilde{h}}{\partial x_{k}} \frac{\partial \tilde{u}_{j}}{\partial x_{k}} .
\end{gathered}
$$

Coefficients $C_{G R S}$ and $C_{G R H}$ are computed through the dynamic coefficient computation described in $\$ 3.3 .1$ and the model name is abbreviated as LES-DGR. 
(c) The mixed model combines the Smagorinsky model with a scale-similarity model which postulates similarity between the SGS and the small resolved scale (Bardina, Ferziger \& Reynolds 1980; Pruett, Sochacki \& Adams 2001). In the scale-similarity model, the flow is additionally filtered with a test filter (here taken to be the same as the grid filter) to obtain the SGS terms which are modelled as follows:

$$
\begin{gathered}
\tau_{i j}-\frac{\delta_{i j}}{3} \tau_{k k}=-2 C_{S M}^{2} \bar{\Delta} \bar{\rho}|S(\bar{\phi})|\left(S_{i j}(\bar{\phi})-\frac{\delta_{i j}}{3} S_{k k}(\bar{\phi})\right)+\bar{\rho}\left(\widetilde{\tilde{u}_{i} \tilde{u}_{j}}-\widetilde{\tilde{u}_{i}} \tilde{u}_{j}\right), \\
\tau_{k k}=C_{Y O} \bar{\Delta}^{2} S^{2}(\bar{\phi})+\bar{\rho}\left(\widetilde{\tilde{u_{i}} \tilde{u}_{i}}-\tilde{\tilde{u}_{i}} \widetilde{\tilde{u}_{i}}\right), \\
\zeta_{j}=-C_{S M}^{2} \frac{\bar{\Delta}^{2} \bar{\rho}|S(\bar{\phi})|}{P r_{s g s}} \frac{\partial \tilde{h}}{\partial x_{j}}+\bar{\rho}\left(\widetilde{\tilde{h} \tilde{u}_{j}}-\tilde{\tilde{h}} \widetilde{\tilde{u}_{j}}\right) .
\end{gathered}
$$

The model name is abbreviated as LES-DMM.

(d) The Clark model (Clark et al. 1979) utilizes the added combination of the Smagorinsky and gradient models to compute the SGS terms

$$
\begin{gathered}
\tau_{i j}-\frac{\delta_{i j}}{3} \tau_{k k}=-2 C_{S M}^{2} \bar{\Delta} \bar{\rho}|S(\bar{\phi})|\left(S_{i j}(\bar{\phi})-\frac{\delta_{i j}}{3} S_{k k}(\bar{\phi})\right)+\frac{1}{12} \bar{\Delta}^{2} \bar{\rho} \frac{\partial \tilde{u}_{i}}{\partial x_{k}} \frac{\partial \tilde{u}_{j}}{\partial x_{k}}, \\
\zeta_{j}=-C_{S M}^{2} \frac{\bar{\Delta}^{2} \bar{\rho}|S(\bar{\phi})|}{P r_{s g s}} \frac{\partial \widetilde{h}}{\partial x_{j}}+\frac{1}{12} \bar{\Delta}^{2} \bar{\rho} \frac{\partial \widetilde{h}}{\partial x_{k}} \frac{\partial \widetilde{u}_{j}}{\partial x_{k}}
\end{gathered}
$$

where $\tau_{k k}$ is computed from (3.13) and $1 / 12$ is the theoretical coefficient of the gradient model obtained by using the Taylor expansion. The model name is abbreviated as LES-DCM.

\subsubsection{Explicitly filtered LES SGS models}

Because of the different mathematical form of the SGS terms in the EFLES formulation, the gradient model described for the conventional LES formulation is no longer derivable here. This is because this model is obtained by using a Taylor series expansion on the SGS stresses; however, in EFLES, the SGS stresses have a different mathematical form than in conventional LES (compare (3.5) and (3.11)) and in EFLES the Taylor series expansion does not simplify and lend itself to a form similar to the gradient model in conventional LES. This limits the SGS models to the Smagorinsky and mixed models.

(a) The Smagorinsky model for the SGS stresses is reformulated by applying an explicit filter to (3.12) to remove the scales below the filter width that are generated by the nonlinearity of the model. Thus,

$$
\tau_{i j}^{e f}-\frac{1}{3} \tau_{k k}^{e f} \delta_{i j}=-\overline{2 C_{S M} \bar{\Delta}^{2} \bar{\rho}|S(\bar{\phi})|\left[S_{i j}(\bar{\phi})-\frac{\delta_{i j}}{3} S_{k k}(\bar{\phi})\right]} .
$$

Using the same procedure, the Yoshizawa model for the trace term is

$$
\tau_{k k}^{e f}=\overline{C_{Y O} \bar{\Delta}^{2} S^{2}(\bar{\phi})}
$$

and the SGS scalar enthalpy flux is modelled as

$$
\zeta_{j}^{e f}=-C_{S M}^{2} \frac{\bar{\Delta}^{2} \bar{\rho}|S(\bar{\phi})|}{\operatorname{Pr}_{s g s}} \frac{\partial \widetilde{h}}{\partial x_{j}} .
$$


The coefficients $C_{S M}$ and $P r_{s g s}$ are dynamically computed as described in $\S 3.3 .1$ using the equivalent $\mathrm{EF}$ quantities defined in \$3.3.2. The model name is abbreviated as EFLES-DSM.

(b) The mixed model in EFLES is obtained by combining the above Smagorinsky model and a scale-similarity term. For the EFLES formulation, the scale-similarity term is evaluated using the actual expression for SGS terms in (3.8)-(3.9) where $\phi$ is replaced by $\bar{\phi}$ :

$$
\begin{aligned}
& \tau_{i j}^{e f}-\frac{\delta_{i j}}{3} \tau_{k k}^{e f}=-\overline{2 C_{S M}^{2} \bar{\Delta} \bar{\rho}|S(\bar{\phi})|\left(S_{i j}(\bar{\phi})-\frac{\delta_{i j}}{3} S_{k k}(\bar{\phi})\right)}+\left(\overline{\bar{\rho} \tilde{u}_{i} \tilde{u}_{j}}-\overline{\bar{\rho} \tilde{\tilde{u}}_{i} \widetilde{\tilde{u}}_{j}}\right), \\
& \tau_{k k}^{e f}=\overline{C_{Y O} \bar{\Delta}^{2} S^{2}(\bar{\phi})}+\left(\overline{\bar{\rho} \tilde{u}_{i} \tilde{u}_{i}}-\overline{\bar{\rho} \tilde{\tilde{u}}_{i}} \widetilde{\tilde{u}}_{i}\right),
\end{aligned}
$$

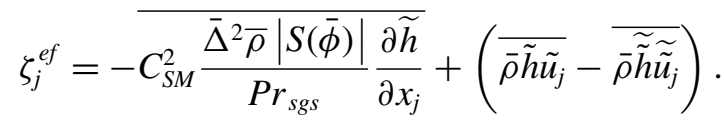

The model name is abbreviated as EFLES-DMM.

\subsection{Model coefficient calculation}

The dynamic model of Germano et al. (1991) is used here to compute the SGS models' coefficients based on the local state of the flow. In the Germano et al. (1991) model, an identity relates the turbulent stresses at grid and test filter levels, leading to an overdetermined set of equations for calculating the SGS-model coefficients. To solve this overdetermination problem, Lilly (1992) proposed utilizing a least-square minimization. Specifically, we follow Martin, Piomelli \& Candler (2000) to compute the coefficients for a compressible flow because the Mach number is larger than the generally accepted value of 0.3 separating the incompressible and compressible regimes. The model of Martin et al. (2000) was developed for conventional LES and is succinctly described in §3.3.1. This model is adapted to EFLES in §3.3.2.

\subsubsection{Conventional LES model coefficient calculation}

The resolved turbulent stress at test filter level can be calculated as

$$
L_{i j}=\left(\frac{\widehat{\rho u_{i} \rho u_{j}}}{\bar{\rho}}\right)-\left(\frac{\widehat{\rho u_{i} \rho u_{j}}}{\widehat{\bar{\rho}}}\right),
$$

where ${ }^{\wedge}$ refers to the filtering at test filter width which, as generally recommended, has twice the width of the grid filter.

For the Smagorinsky model, the Germano et al. (1991) identity relates $L_{i j}$ to modelled stresses at the grid and test filter level as

$$
L_{i j}=C_{S M}^{2} \beta_{i j}-\widehat{C_{S M}^{2} \alpha_{i j}},
$$

where

$$
\begin{aligned}
& \alpha_{i j}=-2 \bar{\Delta}^{2} \bar{\rho}|S(\bar{\phi})|\left[S_{i j}(\bar{\phi})-\frac{\delta_{i j}}{3} S_{k k}(\bar{\phi})\right], \\
& \beta_{i j}=-2 \widehat{\Delta}^{2} \widehat{\bar{\rho}}|S(\widehat{\bar{\phi}})|\left[S_{i j}(\widehat{\bar{\phi}})-\frac{\delta_{i j}}{3} S_{k k}(\widehat{\bar{\phi}})\right] .
\end{aligned}
$$


As explained above, (3.29) represents five equations for one unknown coefficient, and the proposal of Lilly (1992) leads to

$$
\begin{aligned}
C_{S M}^{2} & =\frac{\left\langle L_{i j} M_{i j}\right\rangle}{\left\langle M_{k l} M_{k l}\right\rangle}, \\
M_{i j} & =\beta_{i j}-\widehat{\alpha_{i j}},
\end{aligned}
$$

where \langle\rangle refers to the averaging along the homogeneous planes. Similarly,

$$
C_{Y O}=\frac{\left\langle L_{k k}\right\rangle}{\left\langle P_{m} P_{m}\right\rangle},
$$

where

$$
\begin{gathered}
P_{m}=\beta-\widehat{\alpha}, \\
\alpha=\bar{\Delta}^{2} S^{2}(\bar{\phi}), \quad \beta=\widehat{\Delta}^{2} S^{2}(\widehat{\bar{\phi}}) .
\end{gathered}
$$

Finally, $P r_{s g s}$ is calculated as

$$
\operatorname{Pr}_{s g s}=\frac{C_{S M}^{2}\left\langle T_{k} T_{k}\right\rangle}{\left\langle K_{j} T_{j}\right\rangle},
$$

where

$$
\begin{gathered}
T_{j}=\theta_{j}-\widehat{\psi}_{j}, \\
\theta_{j}=-\widehat{\Delta}^{2} \widehat{\bar{\rho}}|S(\widehat{\bar{\phi}})| \frac{\partial h(\overline{\bar{\phi}})}{\partial x_{j}}, \quad \psi_{j}=\bar{\Delta}^{2} \bar{\rho}|S(\bar{\phi})| \frac{\partial h(\bar{\phi})}{\partial x_{j}}, \\
K_{j}=\left(\frac{\widehat{\overline{\rho u_{j} \rho h}}}{\bar{\rho}}\right)-\left(\frac{\widehat{\widehat{\rho u_{j}}} \widehat{\widehat{\rho h}}}{\widehat{\bar{\rho}}}\right) .
\end{gathered}
$$

For the gradient model, the coefficient $C_{G R S}$ is calculated using

$$
C_{G R S}=\frac{\left\langle L_{i j} Q_{i j}\right\rangle}{\left\langle Q_{i j} Q_{i j}\right\rangle},
$$

where

$$
\begin{gathered}
Q_{i j}=\pi_{i j}-\widehat{\gamma_{i j}} \\
\gamma_{i j}=-\bar{\Delta}^{2} \bar{\rho} \frac{\partial \tilde{u}_{i}}{\partial x_{k}} \frac{\partial \tilde{u}_{j}}{\partial x_{k}}, \quad \pi_{i j}=-\widehat{\bar{\Delta}}^{2} \widehat{\bar{\rho}} \frac{\partial \widetilde{\tilde{u}}_{i}}{\partial x_{k}} \frac{\partial \widetilde{\tilde{u}}_{j}}{\partial x_{k}},
\end{gathered}
$$

where generically

$$
\breve{\varphi}=\widehat{\bar{\rho} \varphi} / \widehat{\hat{\rho}}
$$

refers to the Favre filtering at test filter level. Furthermore, the coefficient $C_{G R H}$ is calculated using

$$
C_{G R H}=\frac{\left\langle K_{j} R_{j}\right\rangle}{\left\langle R_{j} R_{j}\right\rangle},
$$

where

$$
R_{j}=-\widehat{\bar{\Delta}}^{2} \widehat{\bar{\rho}} \frac{\partial \widetilde{\tilde{h}}}{\partial x_{k}} \frac{\partial \widetilde{\tilde{u}_{j}}}{\partial x_{k}}+\bar{\Delta}^{2} \bar{\rho} \frac{\partial \tilde{h}}{\partial x_{k}} \frac{\partial \tilde{u}_{j}}{\partial x_{k}} .
$$


For the dynamic mixed model, $C_{S M}$ is calculated as

$$
C_{S M}^{2}=\frac{\left\langle L_{i j} M_{i j}\right\rangle-\left\langle N_{i j} M_{i j}\right\rangle}{\left\langle M_{k l} M_{k l}\right\rangle}, \quad C_{Y O}=\frac{\left\langle L_{k k}-N_{k k}\right\rangle}{\left\langle P_{m} P_{m}\right\rangle},
$$

with

$$
\begin{aligned}
& N_{i j}=B_{i j}-\widehat{A_{i j}} \text {, }
\end{aligned}
$$

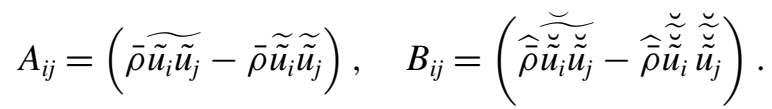

Similarly, $\operatorname{Pr}_{\text {sgs }}$ is calculated as

$$
\operatorname{Pr}_{s g s}=\frac{C_{S M}^{2}\left\langle T_{k} T_{k}\right\rangle}{\left\langle K_{j} T_{j}\right\rangle-\left\langle V_{j} T_{j}\right\rangle}
$$

where

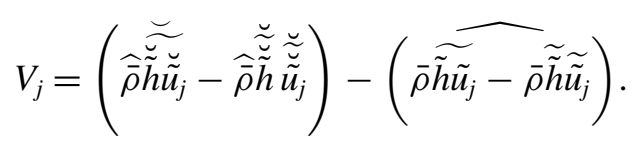

For the dynamic Clark model, the coefficients are calculated using

$$
\begin{gathered}
C_{S M}^{2}=\frac{\left\langle L_{i j} M_{i j}\right\rangle-\left\langle\frac{1}{12} Q_{i j} M_{i j}\right\rangle}{\left\langle M_{k l} M_{k l}\right\rangle}, \quad C_{Y O}=\frac{\left\langle L_{k k}-\frac{1}{12} Q_{k k}\right\rangle}{\left\langle P_{m} P_{m}\right\rangle}, \\
P r_{s g s}=\frac{C_{S M}^{2}\left\langle T_{k} T_{k}\right\rangle}{\left\langle K_{j} T_{j}\right\rangle-\left\langle\frac{1}{12} R_{j} T_{j}\right\rangle} .
\end{gathered}
$$

\subsubsection{Explicitly filtered LES model coefficient calculation}

The EFLES dynamic coefficient calculation follows a parallel methodology, but now with new definitions for the relevant quantities. For example, the resolved turbulent stress at test filter level can be calculated as

$$
L_{i j}^{e f}=\left(\frac{\widehat{\rho u_{i} \rho u_{j}}}{\bar{\rho}}\right)-\left(\frac{\widehat{\widehat{\rho u_{i} \rho u_{j}}}}{\widehat{\bar{\rho}}}\right),
$$

and the equivalent definitions to quantities computed in $\S 3.3 .1$ are

$$
\begin{aligned}
& M_{i j}^{e f}=\widehat{\beta_{i j}}-\widehat{\alpha_{i j}}, \\
& P_{m}^{e f}=\widehat{\beta}-\widehat{\bar{\alpha}}, \\
& K_{j}^{e f}=\left(\frac{\widehat{\overline{\rho u_{j}} \overline{\rho h}}}{\bar{\rho}}\right)-\left(\frac{\widehat{\widehat{\rho u_{j}} \widehat{\widehat{\rho h}}}}{\widehat{\bar{\rho}}}\right) \text {, } \\
& A_{i j}^{e f}=\left(\bar{\rho} \widetilde{\tilde{u}_{i} \tilde{u}_{j}}-\overline{\bar{\rho}} \widetilde{\tilde{u}_{i}} \tilde{\tilde{u}}_{j}\right),
\end{aligned}
$$

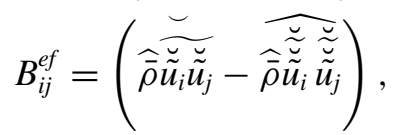


and

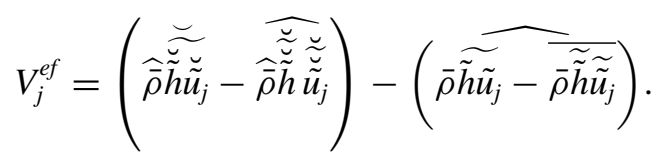

\section{Configuration, initial and boundary conditions}

The physical configuration is that of a temporal shear layer having streamwise $\left(x_{1}\right)$, cross-stream $\left(x_{2}\right)$, and spanwise $\left(x_{3}\right)$ dimensions of $0.6,0.45$ and $0.15 \mathrm{~m}$ respectively. Periodic boundary conditions are used in the $x_{1}$ and $x_{3}$ directions, and an adiabatic slip wall condition (Poinsot \& Lele 1992) is used at the $x_{2}$ boundaries. In LES, this treatment led to instabilities at the $x_{2}$ boundaries and a sponge layer (Israeli \& Orszag 1981) near the boundaries was needed to keep the simulation stable. The gas phase consists of air. To promote transition to turbulence, the velocity field is initially perturbed with homogeneous noise by adding broadband fluctuations similar to that used by Pantano \& Sarkar (2002), the spectra of which are defined by

$$
E(k)=\left(\frac{k}{k_{0}}\right)^{4} \exp \left(-2\left(k / k_{0}\right)^{2}\right)
$$

where $k$ is the wavenumber and $k_{0}$ is the peak wavenumber. The turbulence intensity is $10 \%$; it is added only in the shear layer, and it gradually decreases to zero as one moves away from shear layer along the cross-stream direction. For LES or EFLES, the initial conditions are those of the filtered DNS (FDNS) at $t^{* *} \equiv t \Delta U_{0} / \delta_{\omega, 0}=100$ when the flow transitioned to turbulence as identified from the skewness of the streamwise derivative of the streamwise component of the velocity, which is -0.4 , being well within the necessary range of -0.35 to -0.5 (Lesieur 1997).

The free-stream velocity $U_{0}=M_{c, 0} a_{C, 0}$ is calculated from a specified value of the convective Mach number $M_{c, 0}$ based on the gas initial speed of sound $a_{C, 0}=\sqrt{R T_{0} C_{p} / C_{v}}$ where $T_{0}$ is the initial uniform temperature of the gas at the initial uniform (atmospheric) pressure. The initial vorticity thickness is $\delta_{\omega, 0}=\delta_{\omega}(0)$ where $\delta_{\omega}(t)=\Delta U_{0} /\left(\partial\left\langle u_{1}\right\rangle / \partial x_{2}\right)_{\max }$, with $\Delta U_{0}=2 U_{0}$ being the velocity difference across the layer; the initial mean streamwise velocity has an error-function profile. The specified value of the initial Reynolds number, $\operatorname{Re}_{0}=\rho_{0} \Delta U_{0} \delta_{\omega, 0} / \mu$, where $\rho_{0}$ is the initial gas density, is used to calculate $\mu$. The thermal conductivity is then computed using this value of $\mu$ and (constant) specified value of Prandtl number, $\operatorname{Pr}=0.67$.

\section{Numerical methodology}

For DNS, the nonlinear terms in the governing equation were recast in cubic skew-symmetric form, as proposed by Kennedy \& Gruber (2008), to reduce aliasing error. DNS were performed using a fourth-order explicit Runge-Kutta scheme for temporal integration and an eighth-order central finite differencing scheme for spatial discretization; to remove aliasing errors, a 16th-order filter was used (Kennedy \& Carpenter 1994).

In LES or EFLES, spatial discretization was performed using the fourth-, sixthand eighth-order central schemes proposed by Kennedy \& Carpenter (1994) for the first derivative terms in the differential equations. For the second derivative terms in the LES or EFLES differential equations, fourth-, sixth- and eighth-order narrow stencils proposed by Mattsson \& Nordstrom (2004) were used. The narrow stencil discretization used for second-derivative terms provides better damping for the highest 


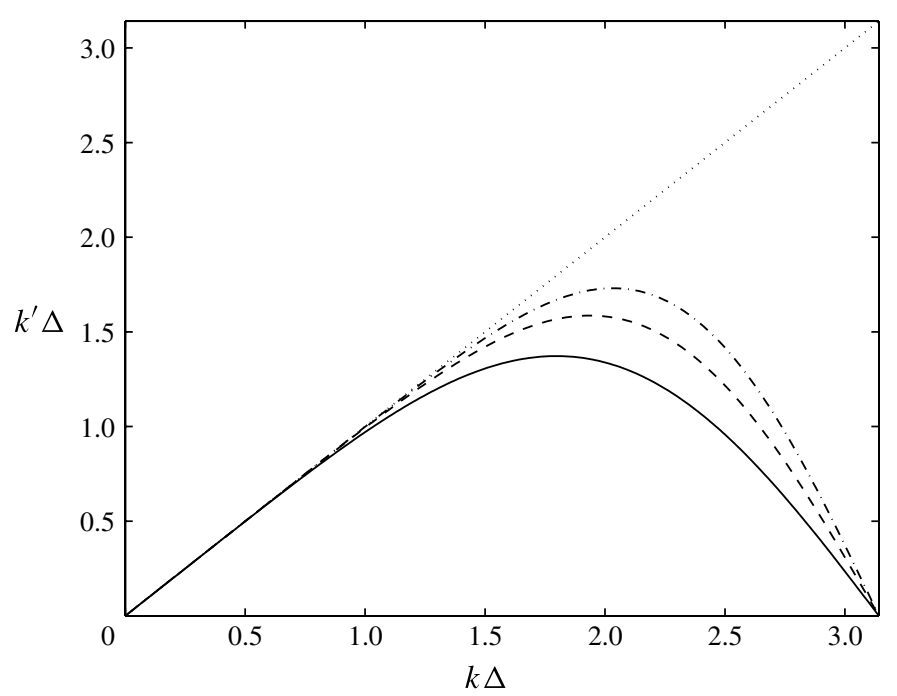

FIGURE 1. Modified wavenumber for fourth-, sixth- and eighth-order central finitedifferencing schemes as a function of non-dimensional wavenumber $k \Delta$. -, fourth-order discretization; ---, sixth-order discretization; ---, eighth-order discretization; $\cdots .$. , exact.

resolved wavenumbers, and thus made it unnecessary to apply the dealiasing filter in LES calculations. The same Runge-Kutta temporal integration scheme utilized in DNS is used for LES and EFLES.

Numerical errors originate in the inability of the spatial discretization to accurately calculate derivatives in the high-wavenumber region. Presented in figure 1 are the modified wavenumber for the fourth-, sixth- and eighth-order central finite differencing schemes used. Compared to low-order schemes, high-order schemes calculate derivatives more accurately for an increased range of wavenumbers. Applying an explicit filter to damp the contribution from the range of wavenumbers where the derivative is inaccurate can prevent the numerical error from affecting the simulation results. The results show that the eighth-order filter effectively damps the inaccurate high-wavenumber contribution. Tables 1 and 2 list several grids (or meshes) employed in the calculations. Illustrated in figure $2(a)$ is the transfer function of the constructed eighth-order filter which shows that on the coarse mesh, where an explicit filter of width twice that of the grid spacing is applied, the explicit filter damps the nondimensional wavenumbers between $\pi / 2$ and $\pi$. On the medium mesh, the filter width is four times the grid spacing and it damps the non-dimensional wavenumbers between $\pi / 4$ and $\pi$. For comparison, in figure $2(b)$, the transfer function of the explicit filter is plotted as a function of wavenumber non-dimensionalized by the grid spacing on the coarse mesh. The plots show that the transfer functions for the explicit filter applied on the coarse, medium and fine meshes coincide, indicating that this filter keeps the same amplitude for a given wavenumber on all meshes. A uniform grid spacing was used in all simulations, particularly to avoid the issue of commutation error due to inhomogenous filters (Pope 2000). The filtered variables $(\bar{f})$ are calculated using

$$
\bar{f}_{i}=a_{0} f_{i}+\sum_{j=1}^{j=M} a_{j}\left(f_{i-j}+f_{i+j}\right)
$$




$\begin{array}{lccccc}\text { Simulation name } & \begin{array}{c}\text { Discretization } \\ \text { order }\end{array} & \bar{\Delta} & \Delta_{L E S} & N_{1} \times N_{2} \times N_{3} & \begin{array}{c}\text { LES/EFLES } \\ \text { grid name }\end{array} \\ \text { DNS-600 } & \text { Eighth } & - & - & 896 \times 672 \times 224 & - \\ \text { LES-DSM-C4-600 } & \text { Fourth } & 4 \Delta_{D N S} & 4 \Delta_{D N S} & 224 \times 168 \times 56 & \mathrm{C} \\ \text { LES-DSM-C6-600 } & \text { Sixth } & 4 \Delta_{D N S} & 4 \Delta_{D N S} & 224 \times 168 \times 56 & \mathrm{C} \\ \text { LES-DSM-C8-600 } & \text { Eighth } & 4 \Delta_{D N S} & 4 \Delta_{D N S} & 224 \times 168 \times 56 & \mathrm{C} \\ \text { LES-DSM-M4-600 } & \text { Fourth } & 2 \Delta_{D N S} & 2 \Delta_{D N S} & 448 \times 336 \times 112 & \mathrm{M} \\ \text { LES-DSM-M6-600 } & \text { Sixth } & 2 \Delta_{D N S} & 2 \Delta_{D N S} & 448 \times 336 \times 112 & \mathrm{M} \\ \text { LES-DSM-M8-600 } & \text { Eighth } & 2 \Delta_{D N S} & 2 \Delta_{D N S} & 448 \times 336 \times 112 & \mathrm{M} \\ \text { EFLES-DSM-C4-600 } & \text { Fourth } & 8 \Delta_{D N S} & 4 \Delta_{D N S} & 224 \times 168 \times 56 & \mathrm{C} \\ \text { EFLES-DSM-C6-600 } & \text { Sixth } & 8 \Delta_{D N S} & 4 \Delta_{D N S} & 224 \times 168 \times 56 & \mathrm{C} \\ \text { EFLES-DSM-C8-600 } & \text { Eighth } & 8 \Delta_{D N S} & 4 \Delta_{D N S} & 224 \times 168 \times 56 & \mathrm{C} \\ \text { EFLES-DSM-M4-600 } & \text { Fourth } & 8 \Delta_{D N S} & 2 \Delta_{D N S} & 448 \times 336 \times 112 & \mathrm{M} \\ \text { EFLES-DSM-M6-600 } & \text { Sixth } & 8 \Delta_{D N S} & 2 \Delta_{D N S} & 448 \times 336 \times 112 & \mathrm{M} \\ \text { EFLES-DSM-M8-600 } & \text { Eighth } & 8 \Delta_{D N S} & 2 \Delta_{D N S} & 448 \times 336 \times 112 & \mathrm{M} \\ \text { EFLES-DSM-F4-600 } & \text { Fourth } & 8 \Delta_{D N S} & \Delta_{D N S} & 896 \times 672 \times 224 & \mathrm{~F} \\ \text { EFLES-DSM-F6-600 } & \text { Sixth } & 8 \Delta_{D N S} & \Delta_{D N S} & 896 \times 672 \times 224 & \mathrm{~F} \\ \text { EFLES-DSM-F8-600 } & \text { Eighth } & 8 \Delta_{D N S} & \Delta_{D N S} & 896 \times 672 \times 224 & \mathrm{~F}\end{array}$

TABLE 1. Abreviations for names of simulations performed using $R e_{0}=600$ (represented by ' 600 ' at the end of each name). The simulations include DNS, LES and EFLES. The listed LES and EFLES were all performed using the dynamic Smagorinsky model (DSM). In the abbreviated name of simulations, ' $C$ ' denotes a coarse grid, ' $M$ ' a medium grid, and ' $F$ ' a fine grid. Each grid label is followed by a number denoting the discretization-order accuracy.

Run

Explicitly filtered LES, coarse mesh Explicitly filtered LES, medium mesh Explicitly filtered LES, fine mesh

$\begin{array}{cc}\bar{\Delta} & \Delta_{L E S} \\ 16 \Delta_{D N S} & 8 \Delta_{D N S} \\ 16 \Delta_{D N S} & 4 \Delta_{D N S} \\ 16 \Delta_{D N S} & 2 \Delta_{D N S}\end{array}$

$N_{1} \times N_{2} \times N_{3}$

$192 \times 192 \times 64$

$384 \times 384 \times 128$

$768 \times 768 \times 256$

TABLE 2. Filter width and grid spacing for LES and EFLES simulations of the mixing layer for which $R e_{0}=1800$.

where $a_{0}, a_{1}, \ldots, a_{j}$ are the weights of the constructed eighth-order filter. Values of coefficients $a_{j}$ for all meshes of table 1 are listed in the Appendix.

\section{Results}

We first present here a succinct description of the DNS database which serves as the basis for the template to be reached in the LES solution. Then, we describe results obtained with the conventional LES formulation and show that the simulations are both grid-spacing and discretization-order dependent. Further, we examine the EFLES predictions and show that grid-spacing independence is obtained for the highest-order spatial discretization on the coarse mesh, while grid-spacing independence is obtained for lower-order spatial discretization only on the medium mesh. Finally, grid-spacing independent simulations allow us to assess the performance of the SGS models without interference of numerical issues. 

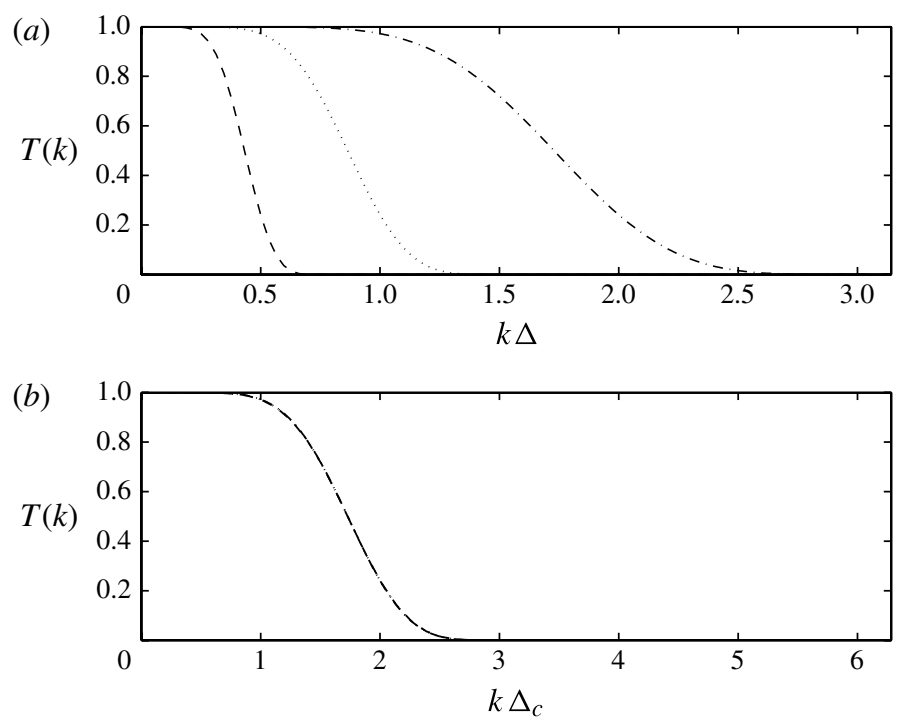

FIGURE 2. Transfer function of the filter applied on: --- , coarse mesh; $\cdots \cdot \cdots$, medium mesh; ---, fine mesh. (a) Transfer function versus non-dimensional wavenumber $k \Delta(b)$ transfer function versus wavenumber non-dimenisionalized by $\Delta_{c}$ (coarse mesh grid spacing).

\subsection{Description of the DNS database}

The baseline DNS database consists of a simulation initiated with $\operatorname{Re}_{0}=600, M_{c, 0}=$ $0.35, T_{C, 0}=375 \mathrm{~K}, \quad \rho_{0}=0.9415 \mathrm{~kg} \mathrm{~m}^{-3}, \Delta U_{0}=271.7 \mathrm{~m} \mathrm{~s}^{-1}$ and $\delta_{\omega, 0}=6.667 \times$ $10^{-3} \mathrm{~m}$. The grid $N_{1} \times N_{2} \times N_{3}$ is $896 \times 672 \times 224$, and the ratio of the grid spacing to the Kolmogorov scale is $\Delta_{D N S} / \eta_{K}=1.5$. At $t^{* *}=0, \delta_{m, 0} / \delta_{\omega, 0}=0.22475$, where

$$
\delta_{m}=\frac{1}{\rho_{0} \Delta U_{0}^{2}} \int_{-L_{2}}^{L_{2}} \rho\left(0.5 \Delta U_{0}-\frac{\left\langle\bar{\rho} \widetilde{u}_{1}\right\rangle}{\langle\bar{\rho}\rangle}\right)\left(0.5 \Delta U_{0}+\frac{\left\langle\bar{\rho} \widetilde{u}_{1}\right\rangle}{\langle\bar{\rho}\rangle}\right) \mathrm{d} x_{2}
$$

is the momentum thickness which measures the growth of the mixing layer. The layer achieves self-similarity at $t^{* *}=70$ when the growth of $\delta_{m}$ becomes linear. The computation is conducted up to $t^{* *}=250$ at which time station $\delta_{m} / \delta_{\omega, 0}=3.3453$ and $\operatorname{Re}_{m} \equiv \rho_{0} \Delta U_{0} \delta_{m} / \mu=2007.2$.

A DNS database was also created for $R e_{0}=1800, M_{c, 0}=0.35, T_{C, 0}=375 \mathrm{~K}, \rho_{0}=$ $0.9415 \mathrm{~kg} \mathrm{~m}^{-3}, \Delta U_{0}=271.7 \mathrm{~m} \mathrm{~s}^{-1}$ and $\delta_{\omega, 0}=6.667 \times 10^{-3} \mathrm{~m}$. The DNS for this higher Reynolds number utilized a grid with $1536 \times 1536 \times 512$ nodes, and the ratio of the grid spacing to the Kolmogorov scale is $\Delta_{D N S} / \eta_{K}=2.0$. The layer achieves self-similarity at $t^{* *}=60$ and the computation is conducted up to $t^{* *}=250$ at which time station $\delta_{m} / \delta_{\omega, 0}=3.5584$ and $R e_{m}=6405$.

\subsection{Acronyms denoting the simulations}

Due to the large number of simulations (2 DNS, 30 LES and 31 EFLES), it was found useful to use abbreviations for denoting the name of each simulation. An example is provided in table 1 for the DNS, all LES-DSM as defined in $\$ 3.2 .1$ and all EFLES-DSM as defined in $\S 3.2 .2$; these simulations are for $R e_{0}=600$. The table also provides information on the order of discretization, filter size and resolution. For other than DNS, in the following, the name of each simulation reflects whether it is LES 
or EFLES, the SGS model used (DSM, DMM, DGR or DCM), the grid spacing (C: coarse; M: medium; F: fine; the $\mathrm{C}, \mathrm{M}$ and $\mathrm{F}$ grid spacings are fixed according to the $R e_{0}$ value) and the order of discretization (fourth, sixth or eighth). The $R e_{0}$ value used in the simulations appears at the end of the name of each simulation. For example, the EFLES performed with the DSM model and a medium grid spacing using a sixth-order discretization and where $R e_{0}=1800$ is denoted by EFLES-DSM-M6-1800. Table 2 lists information on $\bar{\Delta}$ and $\Delta_{L E S}$ compared to $\Delta_{D N S}$, and on the grid spacing for all simulations where $R e_{0}=1800$.

\subsection{Quantities for evaluation of LES and EFLES performance}

The performance of various models will be evaluated according to several quantities which we deem important to have LES results matching those provided by the DNS template. Borrowing the terminology used by Geurts \& Frohlich (2002) and Meyers et al. $(2003,2005,2006)$, we call these quantities 'objectives' to indicate that indeed these may be the objectives of a person undertaking LES. The level of performance evaluation becomes more stringent as the user attempts to satisfy an increasing number of objectives in the order listed (akin but not identical to the list provided by Sagaut \& Deck 2009 for LES used in aerodynamics).

(a) Zeroth (i.e. integral) and first-order quantities, such as $\delta_{m}(t)$ and mean flow variables, all of which we consider the minimum requirement to match in any simulation. Reynolds-averaged quantities are obtained by $\left(x_{1}, x_{3}\right)$ plane averaging, and thus they are denoted by \langle\rangle . Favre-averaged quantities are denoted by \langle\rangle$^{F}$ obtained from filtered quantities, so that

$$
\langle\psi\rangle_{L E S}=\langle\bar{\psi}\rangle, \quad\langle\psi\rangle_{L E S}^{F}=\frac{\langle\overline{\rho \psi}\rangle}{\langle\bar{\rho}\rangle} .
$$

(b) Second-order quantities such as Reynolds stresses and spectra. Reynolds fluctuations and Favre fluctuations are calculated as $\psi^{\prime}=\psi-\langle\psi\rangle_{L E S}$ and $\psi^{\prime \prime}=\psi-\langle\psi\rangle_{L E S}^{F}$. Reynolds stresses $R_{i j}$ are defined as

$$
R_{i j}=\frac{\left\langle\bar{\rho} u_{i}^{\prime \prime} u_{j}^{\prime \prime}\right\rangle}{\rho_{0} \Delta U_{0}^{2}} .
$$

Streamwise spectra of turbulent kinetic energy are computed as

$$
E\left(k_{1}\right)=\frac{1}{2}\left(u_{1}\left(k_{1}\right) u_{1}^{*}\left(k_{1}\right)+u_{2}\left(k_{1}\right) u_{2}^{*}\left(k_{1}\right)+u_{3}\left(k_{1}\right) u_{3}^{*}\left(k_{1}\right)\right),
$$

where $u_{1}\left(k_{1}\right), u_{2}\left(k_{1}\right)$ and $u_{3}\left(k_{1}\right)$ are the Fourier transforms taken along the $x_{1}$ direction of velocity fluctuations $u_{1}^{\prime}, u_{2}^{\prime}$ and $u_{3}^{\prime}, k$ labels the wavenumber and the superscript $*$ denotes the complex conjugate. Spanwise spectra of turbulent kinetic energy are computed from

$$
E\left(k_{3}\right)=\frac{1}{2}\left(u_{1}\left(k_{3}\right) u_{1}^{*}\left(k_{3}\right)+u_{2}\left(k_{3}\right) u_{2}^{*}\left(k_{3}\right)+u_{3}\left(k_{3}\right) u_{3}^{*}\left(k_{3}\right)\right),
$$

where $u_{1}\left(k_{3}\right), u_{2}\left(k_{3}\right)$ and $u_{3}\left(k_{3}\right)$ are the Fourier transforms taken along the $x_{3}$ direction of velocity fluctuations $u_{1}^{\prime}, u_{2}^{\prime}$ and $u_{3}^{\prime}$.

Two-point streamwise correlations of the streamwise velocity fluctuation, are defined as, for example,

$$
R_{u 1 u 1}\left(x_{1}\right)=\frac{\left\langle u_{1}^{\prime}\left(x_{1,0}, x_{2,0}, x_{3,0}\right) u_{1}^{\prime}\left(x_{1,0}+x_{1}, x_{2,0}, x_{3,0}\right)\right\rangle}{\left\langle u_{1}^{\prime}\left(x_{1,0}, x_{2,0}, x_{3,0}\right) u_{1}^{\prime}\left(x_{1,0}, x_{2,0}, x_{3,0}\right)\right\rangle},
$$



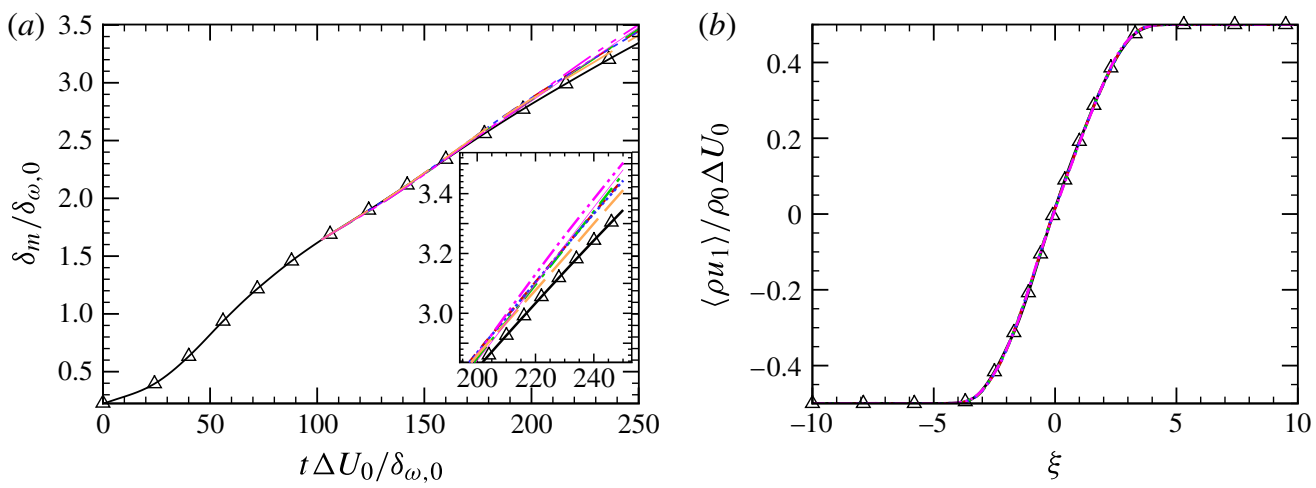

FIGURE 3. (Colour online available at journals.cambridge.org/flm) Mean flow quantities. (a) $\delta_{m}$ timewise evolution and (b) Favre-averaged streamwise velocity. - , DNS; $\triangle$, filtered DNS with $\bar{\Delta}=2 \Delta_{D N S} ;-{ }_{-}$, LES-DSM-M8-600; -- - , LES-DSM-C8-600; ........, LESDSM-M6-600; -..-, LES-DSM-C6-600.

and show approximately how far the fluctuations at one point are correlated with the fluctuations as one moves away from the specified point along the streamwise direction. For example, a non-zero two-point correlation over an extensive streamwise distance implies that the streamwise fluctuations are correlated over a long distance.

Since the mixing layer evolution is self-similar, the statistics are invariant at different instants in time when plotted along the similarity coordinate, $\xi \equiv x_{2} / \delta_{m}(t)$. This fact has been utilized to obtain smoother statistics than when computing them at fixed $t^{* *}$, by temporally averaging the quantities from $t^{* *}=150$ to $t^{* *}=250$, in addition to averaging along the homogeneous directions $\left(x_{1}\right.$ and $\left.x_{3}\right)$.

\subsection{Conventional LES performance}

On trying to compare conventional LES to DNS one encounters a conundrum. Because LES represents the FDNS, it is fitting that the DNS information should be filtered to compare with that from LES. Generally, it is expected that the choice of the filter shape will probably affect the quantitative aspect of the FDNS. However, in conventional LES, the filter shape is not involved in the formulation of the SGS models (see (3.12)-(3.21)); only the filter width appears in SGS models. Thus, comparisons of LES with the FDNS on the LES grid may be subject to controversy because the assessed performance of LES will be filter-shape dependent. As a palliative to this conundrum, we are here comparing LES predictions with both the DNS and the FDNS results, where the FDNS was obtained by using a top-hat filter. As listed in table 1 , in all cases the filter width is $\bar{\Delta}=\Delta_{L E S}$ where $\Delta_{L E S}$ is the LES grid spacing. If LES is grid independent, all simulations should agree independent of the chosen grid spacing. Both $\mathrm{C}$ and $\mathrm{M}$ grids were used in LES.

\subsubsection{The dynamic Smagorinsky model}

The mean quantities are displayed in figure 3 for the DNS, FDNS and LES computations performed with the DSM model for the $\mathrm{C}$ and $\mathrm{M}$ grid spacings listed in table 1 and sixth and eighth-order discretization schemes. The temporal evolution of $\delta_{m} / \delta_{\omega, 0}$ illustrated in figure $3(a)$ indicates that up to the achievement of self-similarity, all curves coincide. During the later times of the layer evolution, while the DNS and 

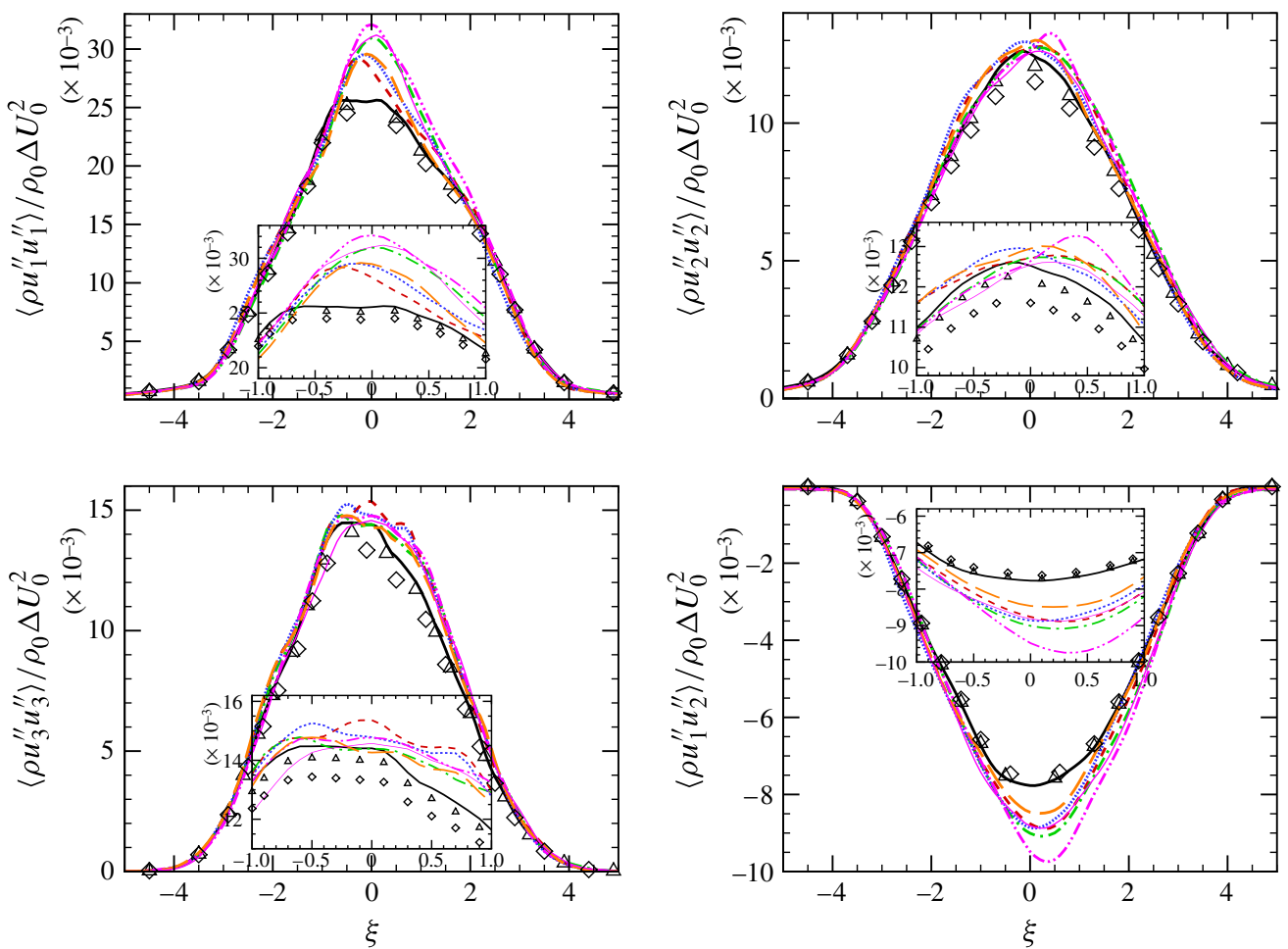

FIGURE 4. (Colour online) Reynolds stress prediction. - DNS; $\triangle$, filtered DNS with $\bar{\Delta}=2 \Delta_{D N S} ; \diamond$, filtered DNS with $\bar{\Delta}=4 \Delta_{D N S} ;-{ }_{-}$, LES-DSM-M8-600; - - , LES-DSMC8-600; ........., LES-DSM-M6-600; -..-, LES-DSM-C6-600; _ _ , LES-DSM-M4-600; , LES-DSM-C4-600.

the FDNS predictions coincide, LES predictions deviate slightly from the FDNS. The Favre-averaged streamwise velocity, $\left\langle\rho u_{1}\right\rangle / \rho_{0} \Delta U_{0}$ exhibited in figure $3(b)$ is plotted versus $\xi$. Clearly, all simulations excellently predict this quantity.

The Reynolds stresses are illustrated versus $\xi$ in figure 4 for all simulations considered in figure 3 and additionally for LES-DSM-C4-600 and LES-DSM-M4600. Except for $\left\langle\rho u_{1}^{\prime \prime} u_{1}^{\prime \prime}\right\rangle / \rho_{0} \Delta U_{0}^{2}$ and for $\left\langle\rho u_{1}^{\prime \prime} u_{2}^{\prime \prime}\right\rangle / \rho_{0} \Delta U_{0}^{2}$, in the central part of the layer both FDNS considerably differ from the DNS. Smaller values are indeed expected for the FDNS since filtering tends to smooth the flow and thus reduce fluctuations. Considering $\left\langle\rho u_{1}^{\prime \prime} u_{1}^{\prime \prime}\right\rangle / \rho_{0} \Delta U_{0}^{2}$, LES with M-grids, independent of the order of discretization, yield considerably more accurate results than those with $\mathrm{C}$ grids. On the C-grid used, not only do the LES results depart from both DNS and FDNS, but increasing the order of discretization does not necessarily improve the predictions, as the fourth-order scheme has higher accuracy than the sixth-order scheme.

The situation is considerably more erratic for $\left\langle\rho u_{2}^{\prime \prime} u_{2}^{\prime \prime}\right\rangle / \rho_{0} \Delta U_{0}^{2}$ where all LES predictions are closer to the DNS than the FDNS. With the exception of the C6 LES which substantially strays from the DNS for $\xi>0$, it is not at all clear that one LES is more faithfully portraying the DNS than the others, although the LES-DSMC4-600 and LES-DSM-M6-600 simulations are closer over the entire $\xi$ range to the DNS. The same state of uncertainty is observed for $\left\langle\rho u_{3}^{\prime \prime} u_{3}^{\prime \prime}\right\rangle / \rho_{0} \Delta U_{0}^{2}$ where, similarly 

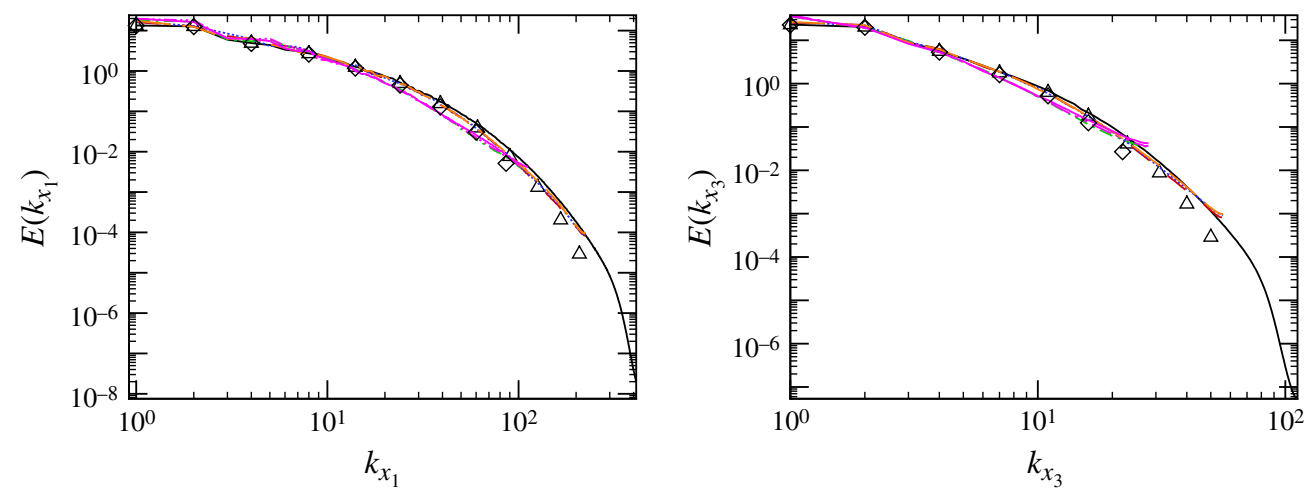

FIGURE 5. (Colour online) Turbulent kinetic energy spectra. - DNS; $\triangle$, filtered DNS with $\bar{\Delta}=2 \Delta_{D N S} ; \diamond$ filtered DNS with $\bar{\Delta}=4 \Delta_{D N S} ;---$, LES-DSM-M8-600; - - - , LES-DSMC8-600; ......... LES-DSM-M6-600; -... , LES-DSM-C6-600; _ _ , LES-DSM-M4-600; , LES-DSM-C4-600.

to the $\left\langle\rho u_{2}^{\prime \prime} u_{2}^{\prime \prime}\right\rangle / \rho_{0} \Delta U_{0}^{2}$ results, the LES predictions are closer to the DNS than to the FDNS. However, for $\left\langle\rho u_{3}^{\prime \prime} u_{3}^{\prime \prime}\right\rangle / \rho_{0} \Delta U_{0}^{2}$ the LES-DSM-M6-600 and LES-DSM-M8-600 are the farthest away from the DNS, closely followed by the LES-DSM-C4-600. The LES-DSM-M4-600 and LES-DSM-C8-600 approximate best the DNS.

A clearer conclusion is obtained by examining $\left\langle\rho u_{1}^{\prime \prime} u_{2}^{\prime \prime}\right\rangle / \rho_{0} \Delta U_{0}^{2}$ where both FDNS coincide with DNS. The LES-DSM-M4-600 is closest to the template whereas the LES-DSM-C6-600 is the least accurate. All other LES have approximately similar quality.

In general, all Reynolds stresses predicted by LES exceed those of the DNS indicating that the DSM as well as all other SGS models (see below) exacerbate the fluctuations at the relatively low wavenumbers which provide the major contributions to these stresses.

The turbulent kinetic energy (TKE) spectra are displayed in figure 5 versus the corresponding wavenumbers encompassing more than two orders of magnitude. The results show that at both $\mathrm{C}$ and $\mathrm{M}$ resolutions, the DSM model predicts slightly more energy than the corresponding FDNS near the largest resolved wavenumber. In fact, these predictions are closer to the DNS results at the largest resolved wavenumber.

Presented in figure 6 is the computed two-point correlation $R_{u 1 u 1}$ in the $x_{1}$ and $x_{3}$ directions. Both FDNS coincide with the DNS in both the $x_{1}$ and $x_{3}$ directions. For $R_{u 1 u 1}\left(x_{1}\right)$, at short separation distances the LES-DSM-M8-600 and LES-DSM-M6-600 agree best with the template, whereas at larger separation distance, LES-DSM-C8-600 yields the more accurate prediction. Unlike $R_{u 1 u 1}\left(x_{1}\right)$, for $R_{u 1 u 1}\left(x_{3}\right)$ LES-DSM-M4-600 and LES-DSM-M8-600 are most accurate overall, with the coarse-grid predictions being the worst. In fact, for the C-grid, $R_{u 1 u 1}\left(x_{3}\right)$ does not reach the null value at large separation distance, a characteristic which we attribute to the large $\bar{\Delta}=4 \Delta_{D N S}$ and the coarse grid used in this case.

Thus, it is clear that there is no consensus as to which numerical scheme or grid spacing provides the best reproduction of the DNS or FDNS from the point of view of all quantities of interest. However, what emerges from these comparisons is that the predictions from the conventional LES are sensitive to both numerical schemes and grid spacing. 

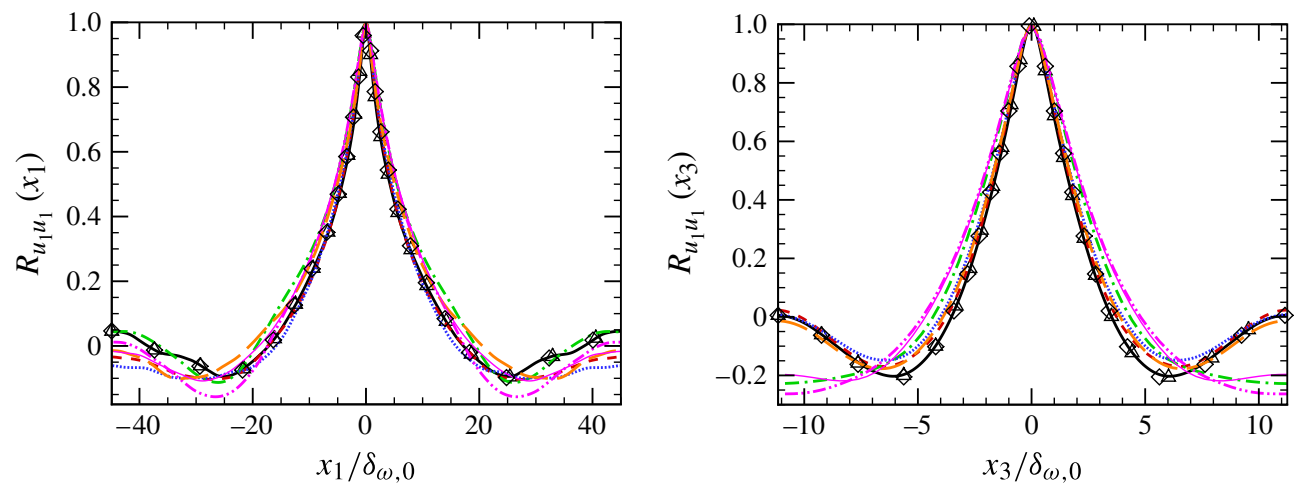

FIgURE 6. (Colour online) Two-point correlation, $R_{u 1 u 1}$, versus $x_{1} / \delta_{\omega, 0}$ and $x_{3} / \delta_{\omega, 0} .-$, DNS; $\triangle$, filtered DNS with $\bar{\Delta}=2 \Delta_{D N S} ; \diamond$, filtered DNS with $\bar{\Delta}=4 \Delta_{D N S}$; --- , LES-DSMM8-600; --=, LES-DSM-C8-600; ........, LES-DSM-M6-600; _..., LES-DSM-C6-600; — — , LES-DSM-M4-600; — , LES-DSM-C4-600.

\subsubsection{The dynamic mixed model}

Mean-quantity predictions emulate for the DMM model those of LES employing the DSM model, and therefore are omitted from the presentation. Because it is symptomatic of the LES quality, only the ability to reproduce the Reynolds stresses will be here examined from the plots illustrated in figure 7 . The width of the test filter used in the simulation was twice the width of the grid filter, as usually recommended.

Similar to the results discussed in $\S 6.4 .1$, for $\left\langle\rho u_{1}^{\prime \prime} u_{1}^{\prime \prime}\right\rangle / \rho_{0} \Delta U_{0}^{2}$ there is a segregation in LES prediction according to the grid spacing; independent of order of discretization, the M-grid LES approximates better the DNS than the C-grid LES. Considering $\left\langle\rho u_{2}^{\prime \prime} u_{2}^{\prime \prime}\right\rangle / \rho_{0} \Delta U_{0}^{2}$, the LES-DMM-M4-600 and LES-DMM-M8-600 agree better with the DNS with LES-DMM-M4-600 being the best, while LES-DMM-M6-600 agrees better with FDNS computed for $\bar{\Delta}=2 \Delta_{D N S}$. The C-grid LES reproduce better the FDNS computed for $\bar{\Delta}=4 \Delta_{D N S}$, with C6 being the most accurate. For $\left\langle\rho u_{3}^{\prime \prime} u_{3}^{\prime \prime}\right\rangle / \rho_{0} \Delta U_{0}^{2}$, the LES-DMM-C6-600 considerably diverges from the FDNS, while the other LES are close to their $\bar{\Delta}$ respective FDNS. For $\left\langle\rho u_{1}^{\prime \prime} u_{2}^{\prime \prime}\right\rangle / \rho_{0} \Delta U_{0}^{2}$, the LESDMM-M6-600 has the best correspondence to the DNS and FDNS, and the LESDMM-C6-600 LES clearly diverges the most from the template.

\subsubsection{The dynamic gradient and dynamic Clark models}

Results for the Reynolds stresses are discussed here, for completeness, but figures are not shown, for brevity. For $\left\langle\rho u_{1}^{\prime \prime} u_{1}^{\prime \prime}\right\rangle / \rho_{0} \Delta U_{0}^{2}$, all DGR model LES considerably overpredict the DNS and FDNS results. The prediction on the M-grid is closer to the DNS, the DCM model provides the closest estimate for LES-DCM-M4-600, and the predictions furthest from the templates are from LES-DCM-C4-600 and LES-DCM-C6-600, both of which provide overestimates. Considering $\left\langle\rho u_{2}^{\prime \prime} u_{2}^{\prime \prime}\right\rangle / \rho_{0} \Delta U_{0}^{2}$, computations with LES-DGR display a clear segregation in terms of LES performance, with the C-grid LES agreeing better with the FDNS and the M-grid LES duplicating better the DNS; the higher-order schemes show better agreement with the DNS or FDNS than the lower-order schemes. Finally, when using the DCM model, LESDCM-C4-600 and LES-DCM-M4-600 deviate the most from the template and produce overestimates. For $\left\langle\rho u_{3}^{\prime \prime} u_{3}^{\prime \prime}\right\rangle / \rho_{0} \Delta U_{0}^{2}$, all DGR simulations emulate the behaviour seen for $\left\langle\rho u_{2}^{\prime \prime} u_{2}^{\prime \prime}\right\rangle / \rho_{0} \Delta U_{0}^{2}$, and when using the DCM model there is no single LES that 

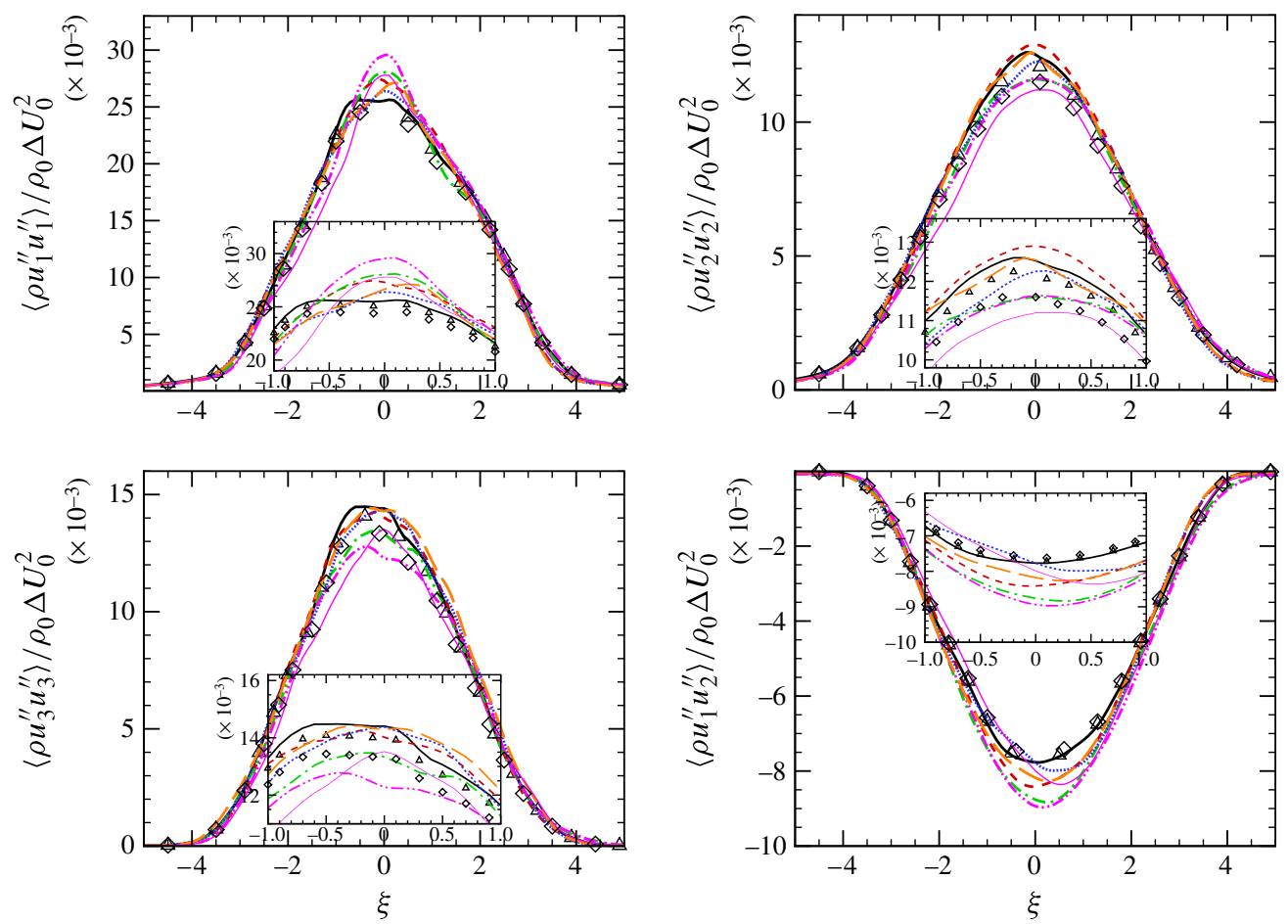

FIGURE 7. (Colour online) Reynolds stress prediction. - DNS; $\triangle$, filtered DNS with $\bar{\Delta}=$ $2 \Delta_{D N S} ; \diamond$, filtered DNS with $\bar{\Delta}=4 \Delta_{D N S} ;---$, LES-DMM-M8-600; - - - , LES-DMMC8-600; ........., LES-DMM-M6-600; -..., LES-DMM-C6-600; — — , LES-DMM-M4600 ; , LES-DMM-C4-600.

stands out as being best. Finally, when examining $\left\langle\rho u_{1}^{\prime \prime} u_{2}^{\prime \prime}\right\rangle / \rho_{0} \Delta U_{0}^{2}$, the C-grid LESDCM are the worst predictors for this quantity.

\subsection{Explicitly filtered LES performance}

The scrutiny of the conventional LES performance revealed several deficiencies, some of which are well-known (Pope 2000, 2004) but have not been examined as systematically as above. For example, the lack of grid independence calls into question the 'completeness' of conventional LES (Pope 2000). Pope (2004) proposes strategies to render LES 'complete'. We observe here that most models tend to overpredict the Reynolds stresses, with the C-grid showing the largest overprediction, while predictions from neither the M-grid nor the C-grid matched the FDNS. This indicates that the reliability of conventional LES to agree with the expected FDNS is poor. Moreover, the dependence of the results on the order of discretization adds to the lack of confidence in the predictions. Since small scales are affected by numerical errors, we inquire here whether explicitly filtering the nonlinear terms in the conservation equations that are responsible for the generation of small scales can mitigate the problem.

Because in EFLES the filter explicitly appears in the formulation, there is no controversy regarding the fact that the same filter shape must be used for FDNS and SGS term computation. Similarly, the filter width used to compute the FDNS is also used in EFLES. Moreover, as listed in table $1, \bar{\Delta}$ is independent of the grid resolution 

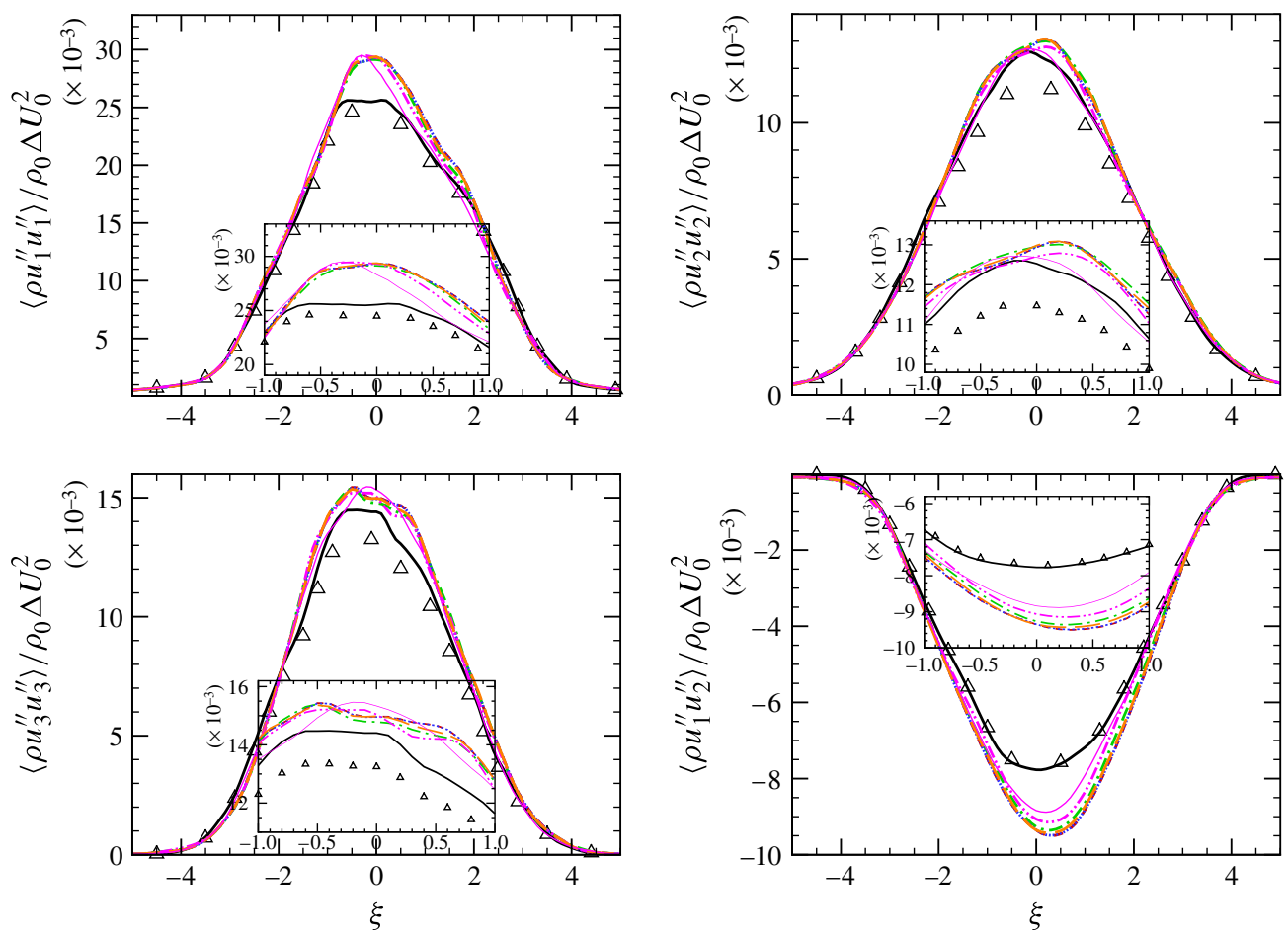

FIGURE 8. (Colour online) Reynolds stress prediction. - DNS; $\triangle$, filtered DNS; - - - , EFLES-DSM-M8-600; ---, EFLES-DSM-C8-600; EFLES-DSM-C6-600; — _ , EFLES-DSM-M4-600; EFLES-DSM-M6-600; -..-, , EFLES-DSM-C4-600.

for EFLES. Thus, for the F-mesh, $\bar{\Delta}$ has a value which is eight times the grid spacing used in LES; for the M-mesh resolution, $\bar{\Delta}$ has a value which is four times the grid spacing used in LES; and for the C-grid resolution, the $\bar{\Delta}$ value is twice the grid spacing used in LES. The idea is that explicit filtering should remove scales smaller than $\bar{\Delta}$ and that this action may induce grid independence for the simulation, in which case refining the grid successively should yield the same result for two consecutive grids.

\subsubsection{The dynamic Smagorinsky model}

Results from EFLES-DSM are illustrated in figures 8-10. Considering the Reynolds stresses, the most crucial change from the conventional LES is that all EFLES predictions nearly coincide, although complete agreement among them is still not obtained. As for LES, the EFLES predictions overestimate the Reynolds stresses. For $\left\langle\rho u_{1}^{\prime \prime} u_{1}^{\prime \prime}\right\rangle / \rho_{0} \Delta U_{0}^{2}$, with the exception of EFLES-DSM-C4-600 and to a much smaller extent EFDLES-DSM-C6-600, all predictions agree, although they all overpredict the filtered DNS. For $\left\langle\rho u_{2}^{\prime \prime} u_{2}^{\prime \prime}\right\rangle / \rho_{0} \Delta U_{0}^{2}$, the spread among predictions is also very small, although those from EFLES-DSM-C6-600 differ somewhat from the other results, and the EFLES-DSM-C4-600, which departs the most from the other four EFLES, agrees the best with the DNS, but not the FDNS. This is no longer the case for $\left\langle\rho u_{3}^{\prime \prime} u_{3}^{\prime \prime}\right\rangle / \rho_{0} \Delta U_{0}^{2}$ where the EFLES-DSM-C4-600 departs the most from the filtered DNS, with all other EFLES closely bunched. Compared to figure 4 obtained with LES, figure 8 shows that the spread of the EFLES predictions has substantially 

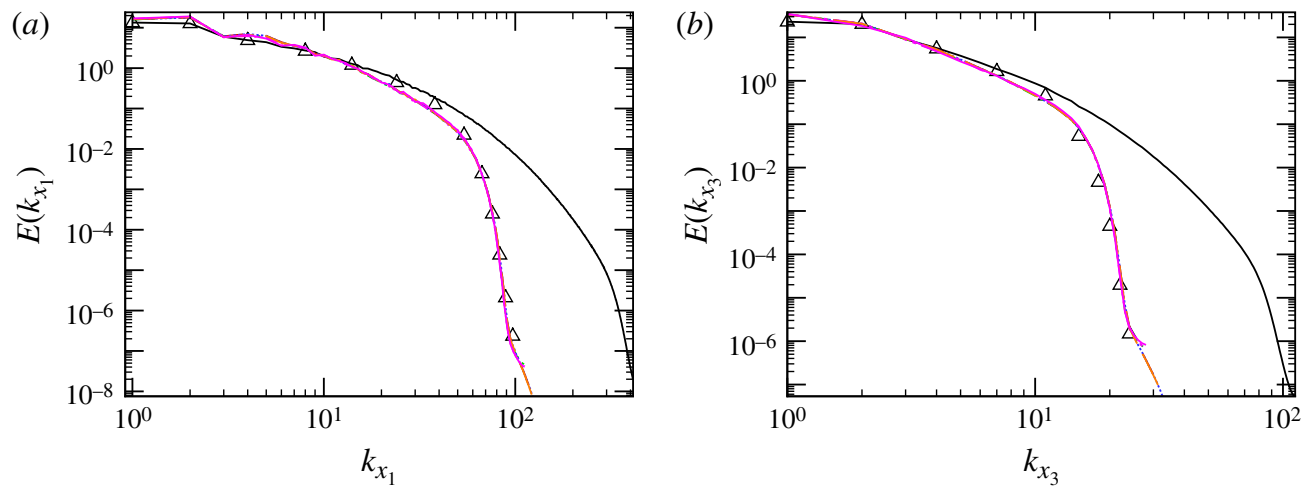

FIGURE 9. (Colour online) Turbulent kinetic energy spectra. - DNS; $\triangle$, filtered DNS; - - - , EFLES-DSM-M8-600; --_, EFLES-DSM-C8-600; ........., EFLES-DSM-M6-600; -..-, EFLES-DSM-C6-600; ——, EFLES-DSM-M4-600; — , EFLES-DSM-C4600.
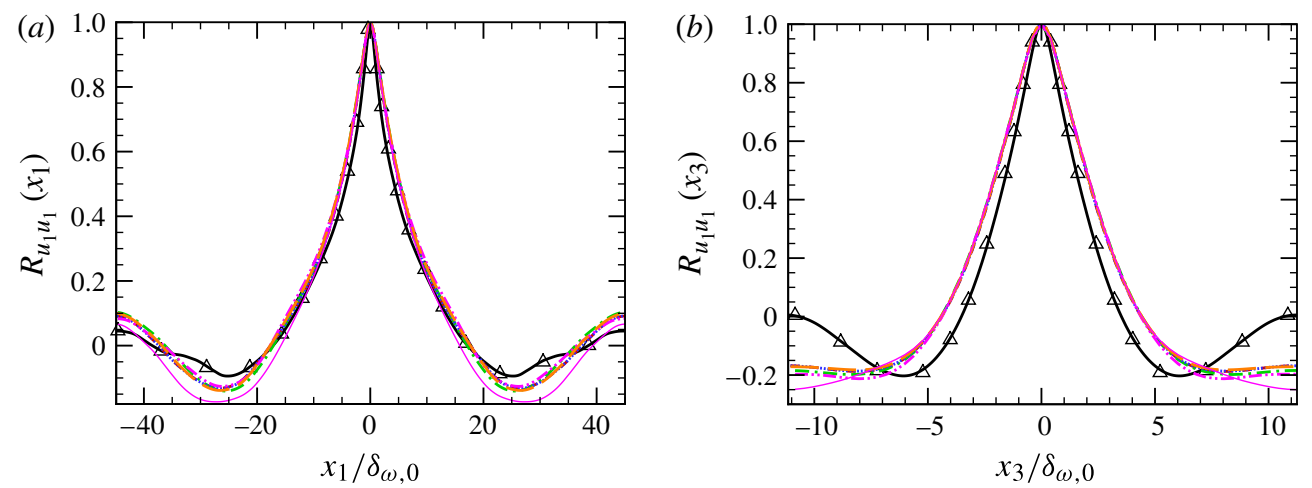

FIGURE 10. (Colour online) Two-point correlations prediction. - , DNS; $\triangle$ filtered DNS; - - - , EFLES-DSM-M8-600; -- - , EFLES-DSM-C8-600; -..-, EFLES-DSM-C6-600; —— , EFLES-DSM-M4-600; EFLES-DSM-M6-600; 600.

narrowed and is only apparent for the C-grid simulations with the exception of EFLES-DSM-C8-600 for which results nearly coincide with those of the EFLESDSM-M8-600 simulation.

The most substantial difference between LES and EFLES is in the TKE spectra displayed in figure 9. All EFLES follow the FDNS, as they should, rather than the DNS as was the case for the results discussed in $\S 6.4 .1$. The assumptions that it is reasonable to filter the convective term in the continuity equation and that the term expressing the work due to resolved stresses in the energy equation does not require explicit filtering in this particular case (see § 3.1.2) are thus justified. Also, all spectra coincide, which means that explicit filtering removes scales smaller than that of the filter, bringing the EFLES in concert with the concept of large-eddy simulations as indeed intended.

For $R_{u 1 u 1}\left(x_{1}\right)$ and $R_{u 1 u 1}\left(x_{3}\right)$ displayed in figure 10, all EFLES with the exception of EFLES-DSM-C4-600 coincide. When examining $R_{u 1 u 1}\left(x_{1}\right)$, it is clear that at short 

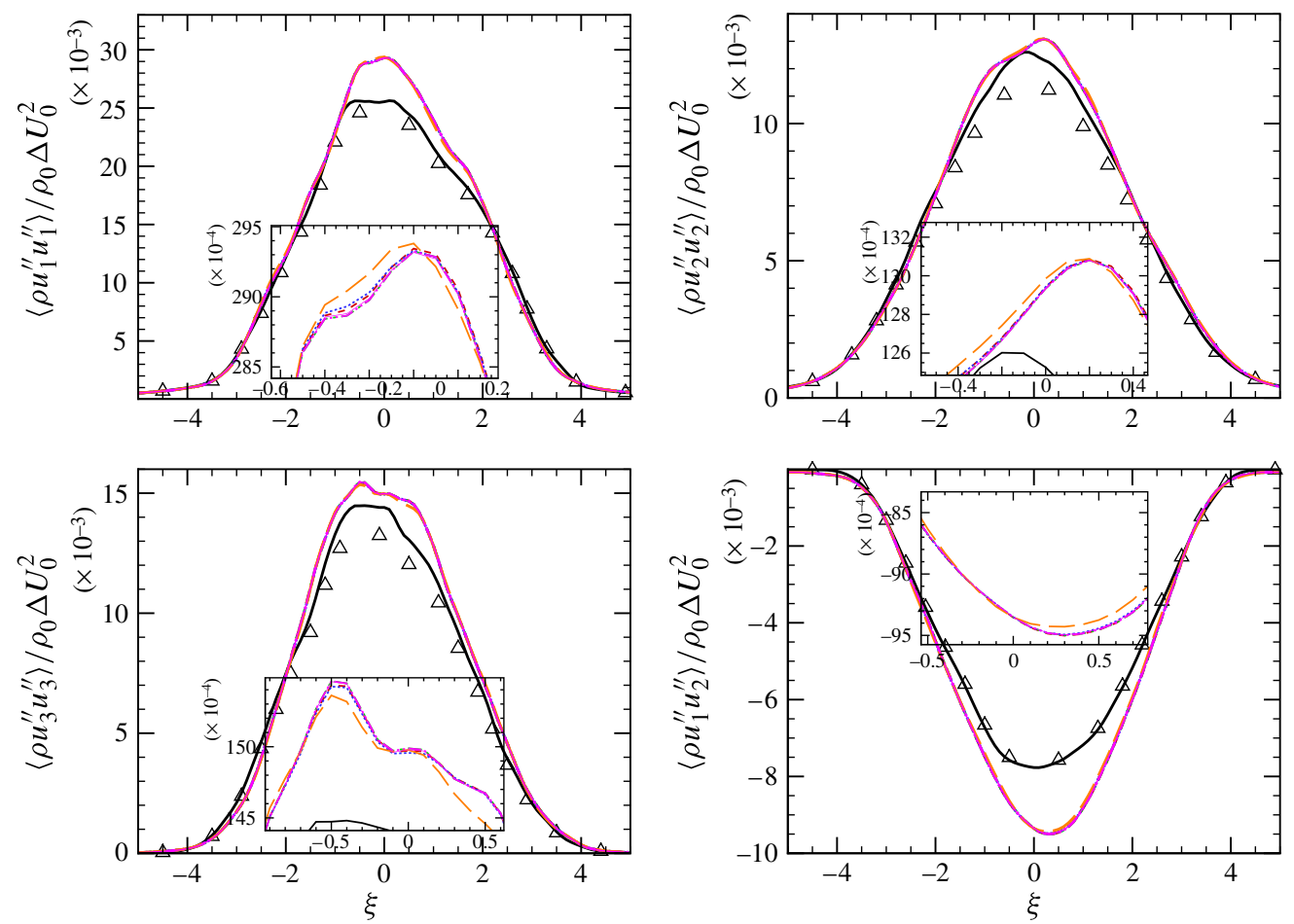

FIGURE 11. (Colour online) Reynolds stress prediction. - DNS; $\triangle$, filtered DNS; - - - , EFLES-DSM-M8-600; - - - , EFLES-DSM-F8-600; ........, EFLES-DSM-M6-600; -..-, EFLES-DSM-F6-600; , EFLES-DSM-M4-600; , EFLES-DSM-F4-600.

separation distances, the EFLES predicts it accurately, but as the location of interest moves away to large separation distance, first an underestimate, then an overestimate occurs. $R_{u 1 u 1}\left(x_{3}\right)$ is not as well reproduced as $R_{u 1 u 1}\left(x_{1}\right)$, but similarly to $R_{u 1 u 1}\left(x_{1}\right)$ all EFLES with the exception of EFLES-DSM-C4-600 give very similar predictions. To summarize, the M-mesh EFLES yield discretization-order-independent results whereas the C-mesh EFLES predictions are discretization-order dependent. With the eighthorder discretization scheme, the results are also grid-spacing independent. Regarding accuracy, it is noticeable that for all grids $R_{u 1 u 1}\left(x_{3}\right)$ does not reach the null value at large separation distance, a fact which is conjectured to result from the large filter width used $\left(\bar{\Delta}=8 \Delta_{D N S}\right)$ that exceeds the value used in the conventional LES C-grid computations. Since LES is an approximation of the FDNS, it is clear that not all aspects of the FDNS may be recoverable and that the LES accuracy may decrease with increasing filter width.

Given that refining the grid by halving the grid spacing did not yield grid convergence of the results for the sixth- and fourth-order discretizations, we investigate the effect of further refining the grid for all the discretization schemes. Results from EFLES using the DSM model on the M- and F-meshes are illustrated in figure 11. All these results coincide, except for negligible difference exhibited by EFLES-DSMM4-600, showing that a grid-spacing and discretization-order independent solution has been obtained by explicitly filtering the nonlinear terms. For the eighth-order scheme, results from all three meshes ( $\mathrm{C}, \mathrm{M}$ and $\mathrm{F}$ ) coincide indicating that the solution obtained on the $\mathrm{C}$-mesh itself is the grid-spacing-independent solution. For the 

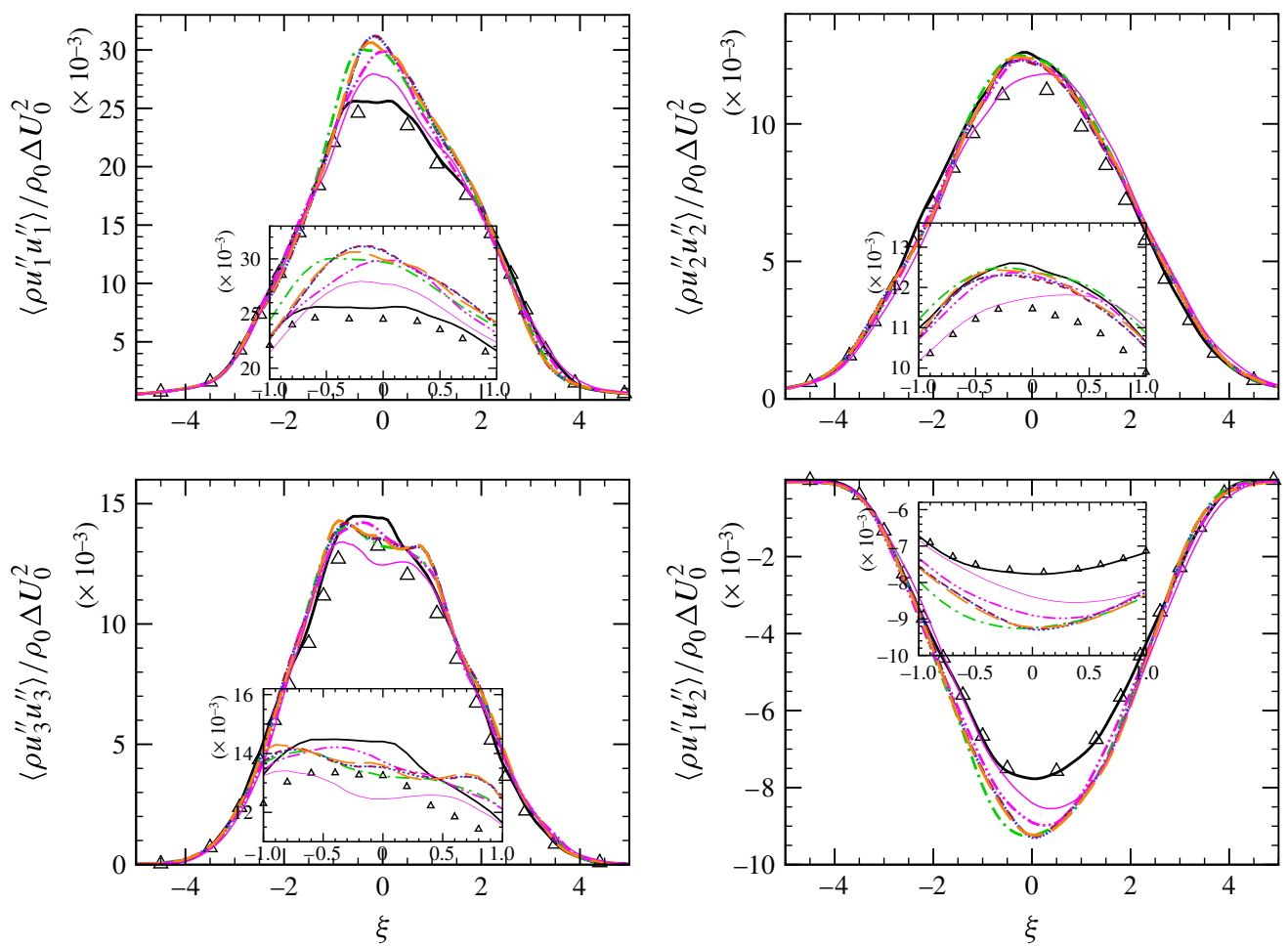

FIGURE 12. (Colour online) Reynolds stress prediction. - , DNS; $\triangle$, filtered DNS; - - - , EFLES-DMM-M8-600; ---, EFLES-DMM-C8-600; ........, EFLES-DMM-M6-600; -..-, EFLES-DMM-C6-600; _ _ , EFLES-DMM-M4-600; — , EFLES-DMM-C4-600.

fourth- and sixth-order discretization schemes, only results from the M- and F-meshes coincide, indicating that the filter-width to grid-spacing ratio used with the $\mathrm{C}$-mesh is not sufficient to guarantee a grid-spacing-independent solution. These results indicate that for the eighth-order scheme, a filter-width to grid-spacing ratio of two is sufficient to yield a grid-spacing-independent solution, while for the fourth- and sixth-order discretization schemes, a filter-width to grid-spacing ratio of four is needed to yield a grid-spacing-independent solution. It is noteworthy that the fine grid utilized in the EFLES is the same as that utilized in DNS. On this DNS grid, the EFLES solution converges to a FDNS (with $\bar{\Delta}=8 \Delta_{D N S}$ ), thus showing that the asymptotic solution of the EFLES approach is not the DNS flow field, unlike in the conventional LES.

\subsubsection{The dynamic mixed model}

Figure 12 shows the Reynolds stress prediction from EFLES using the dynamic mixed model. The predictions are more dependent on the grid and the order of discretization than the predictions obtained with the EFLES-DSM model. All four components of Reynolds stresses obtained utilizing the three M-mesh calculations coincide, indicating that for this model and at this resolution, there is independence of the order of the numerical scheme. For $\left\langle\rho u_{1}^{\prime \prime} u_{1}^{\prime \prime}\right\rangle / \rho_{0} \Delta U_{0}^{2}$, the predictions from EFLESDMM-C8-600 closely approach those of EFLES-DMM-M8-600 while those from EFLES-DMM-C6-600 and EFLES-DMM-C4-600 considerably deviate from the Mmesh predictions. Except for EFLES-DMM-C4-600, the prediction of $\left\langle\rho u_{1}^{\prime \prime} u_{2}^{\prime \prime}\right\rangle / \rho_{0} \Delta U_{0}^{2}$ by all the other simulations show good agreement. Both $\left\langle\rho u_{2}^{\prime \prime} u_{2}^{\prime \prime}\right\rangle / \rho_{0} \Delta U_{0}^{2}$ and 

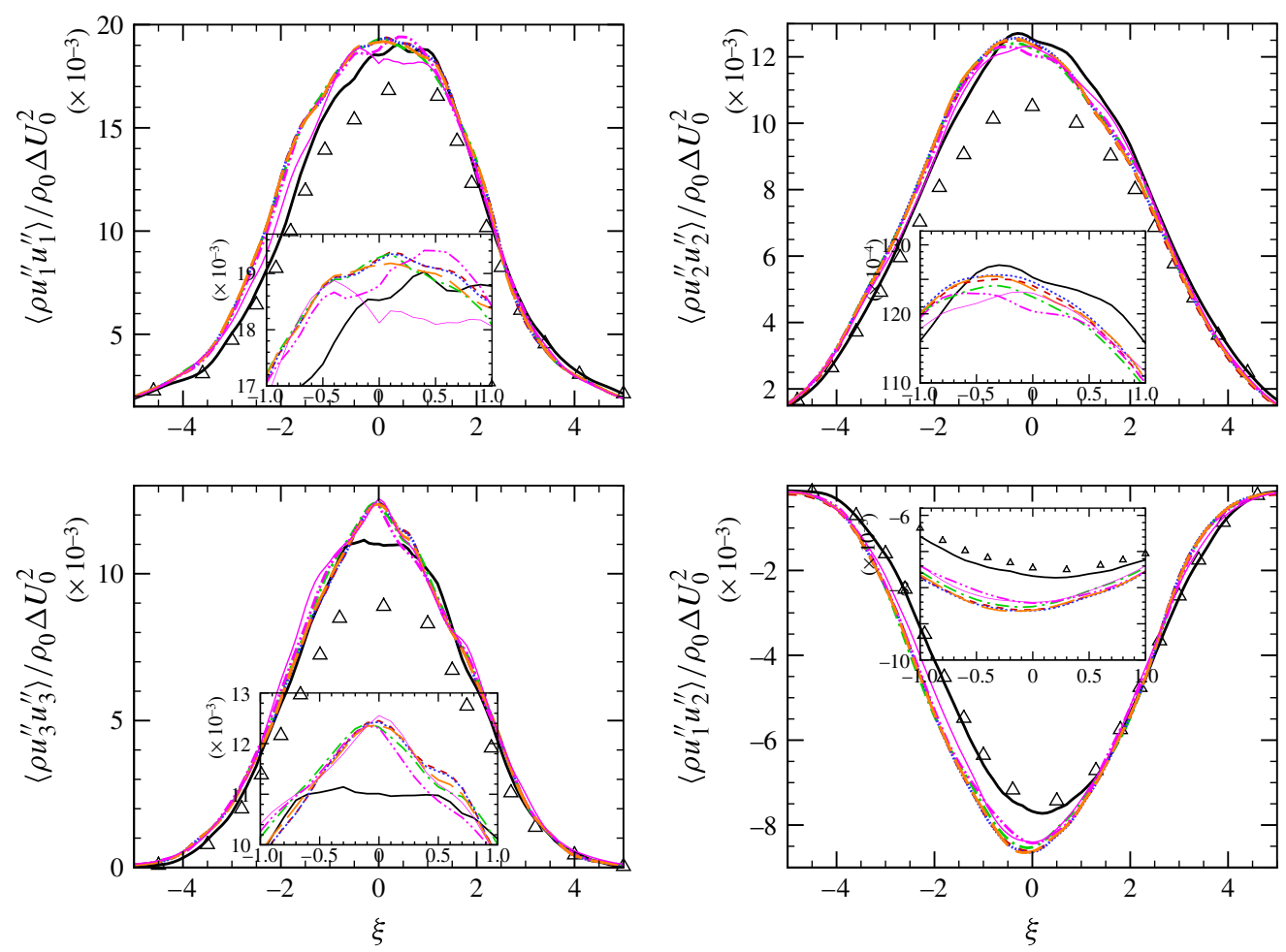

FIGURE 13. (Colour online) Reynolds stress prediction for $R e_{0}=1800$ case. - DNS; $\triangle$, filtered DNS; - - - , EFLES-DSM-M8-1800; - - - , EFLES-DSM-C8-1800; ........., EFLESDSM-M6-1800; -..-, EFLES-DSM-C6-1800; — — , EFLES-DSM-M4-1800; EFLES-DSM-C4-1800.

$\left\langle\rho u_{3}^{\prime \prime} u_{3}^{\prime \prime}\right\rangle / \rho_{0} \Delta U_{0}^{2}$ show the same trend as for $\left\langle\rho u_{1}^{\prime \prime} u_{1}^{\prime \prime}\right\rangle / \rho_{0} \Delta U_{0}^{2}$, i.e. EFLES-DMM-C4600 and EFLES-DMM-C6-600 results deviate from the predictions of the M-mesh calculations which all coincide, and also differ from the $\mathrm{C}$-mesh results obtained with EFLES-DMM-C8-600 which agree with the M-grid predictions over the $\xi>0$ part of the domain. These results also show the same trend as the EFLES-DSM results.

\subsubsection{Simulation with $R_{0}=1800$ using the dynamic Smagorinsky model}

We showed above that with explicit filtering, a grid-spacing- and discretization-orderindependent solution was obtained for a mixing layer initiated with $R e_{0}=600$. By initiating simulations with $R e_{0}=1800$, we here investigate two topics: (i) whether explicit filtering yields similar grid-spacing- and discretization-order-independent results at higher $R e_{0}$, and (ii) whether the necessarily dissipative effect of the explicit filter provides sufficient dissipation, in which case the SGS model would become unnecessary in the EFLES formulation.

To study the first topic, we performed simulations on three meshes, as shown in table 2. Unlike for $R e_{0}=600$, the F-mesh here is coarser than the DNS mesh. Results from EFLES-DSM with $R e_{0}=1800$ are illustrated in figure 13. Similar to the $R e_{0}=600$ case, the Reynolds stresses are overpredicted in all the EFLES simulations. Consistent with the trend observed in the $R e_{0}=600$ case, predictions from EFLESDSM-M4-1800, EFLES-DSM-M6-1800, EFLES-DSM-M8-1800 and EFLES-DSM-C81800 bunch together whereas the prediction from EFLES-DSM-C6-1800 and EFLES- 

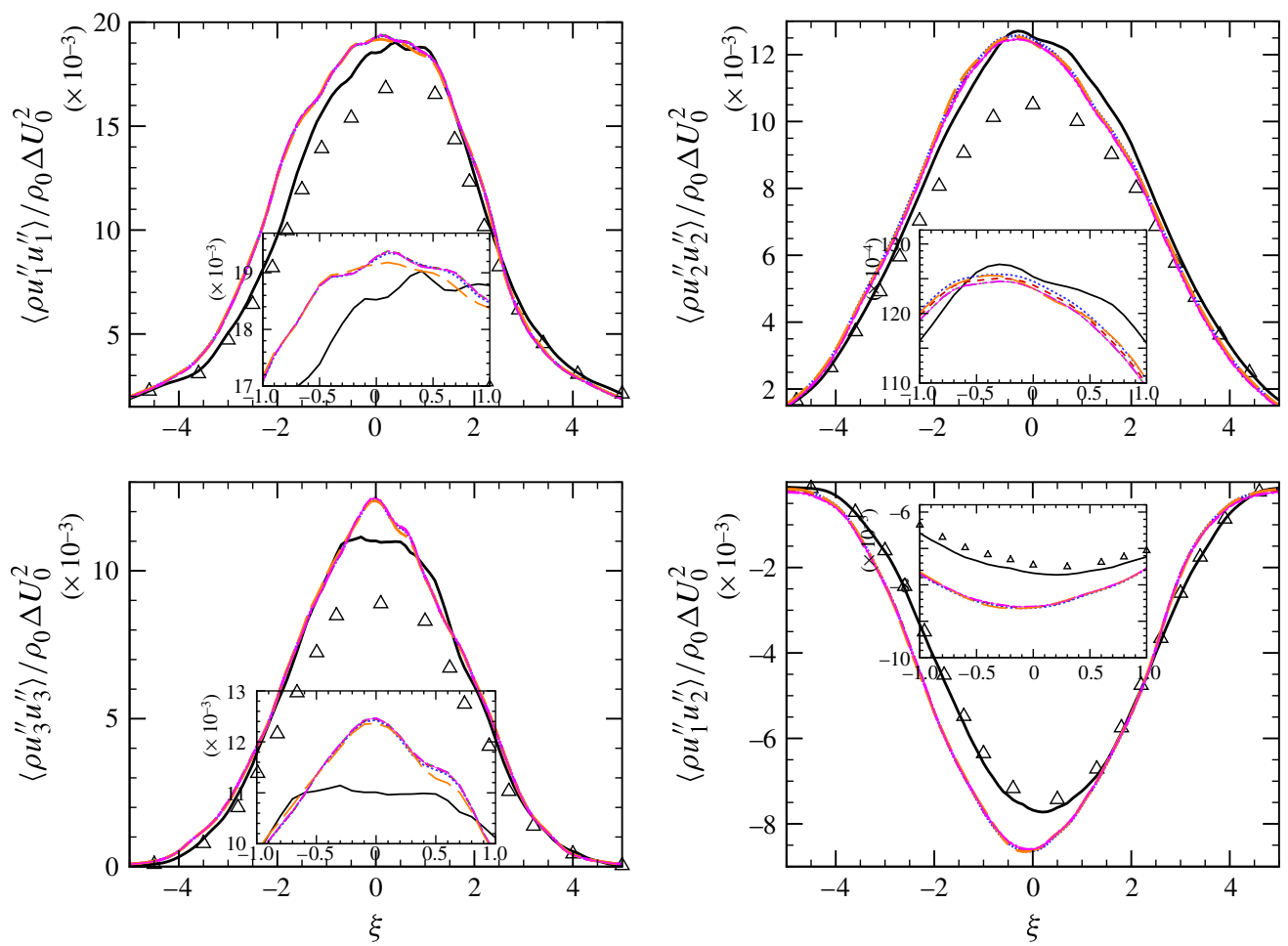

FIGURE 14. (Colour online) Reynolds stress prediction for $R e_{0}=1800$ case. - , DNS; $\triangle$, filtered DNS; - - - , EFLES-DSM-M8-1800; ---, EFLES-DSM-F8-1800; ........., EFLESDSM-M6-1800; -..-, EFLES-DSM-F6-1800; _ _ , EFLES-DSM-M4-1800; _ _, EFLES-DSM-F4-1800.
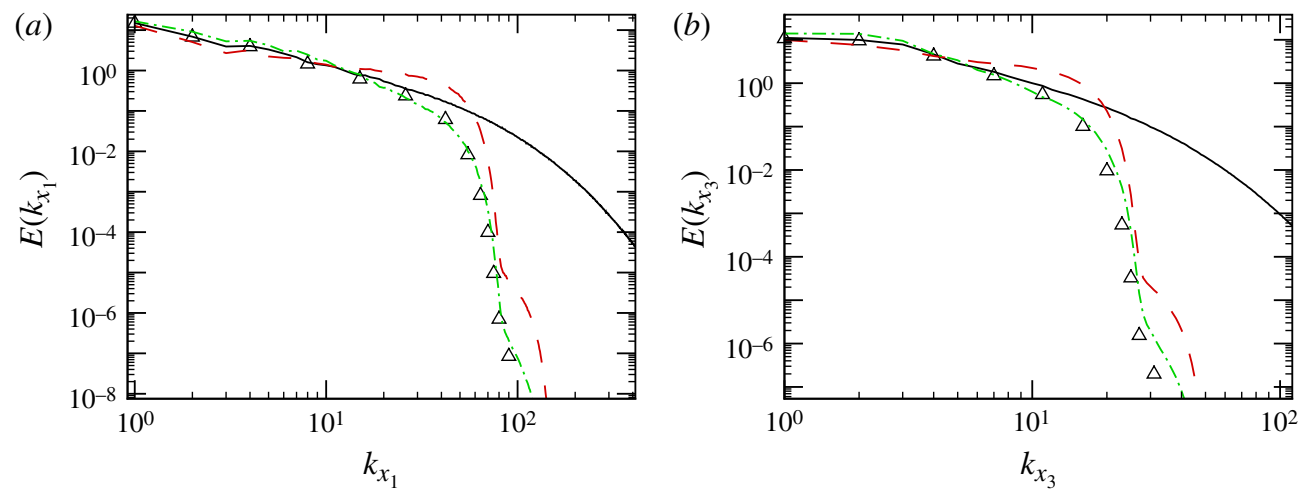

FIGURE 15. (Colour online) Turbulent kinetic energy spectra. - , DNS; $\triangle$, filtered DNS; - - - , EFLES-NM-M8-1800; - - , EFLES-DSM-M8-1800.

DSM-C4-1800 differ. Results on the F- and M-meshes are displayed in figure 14, showing that the results obtained on the F- and M-meshes coincide.

To study the second topic, we performed a no-SGS-model (NM) EFLES, EFLESNM-F8-1800, and compared it to EFLES-DSM-F8-1800. Figure 15 shows the TKE 

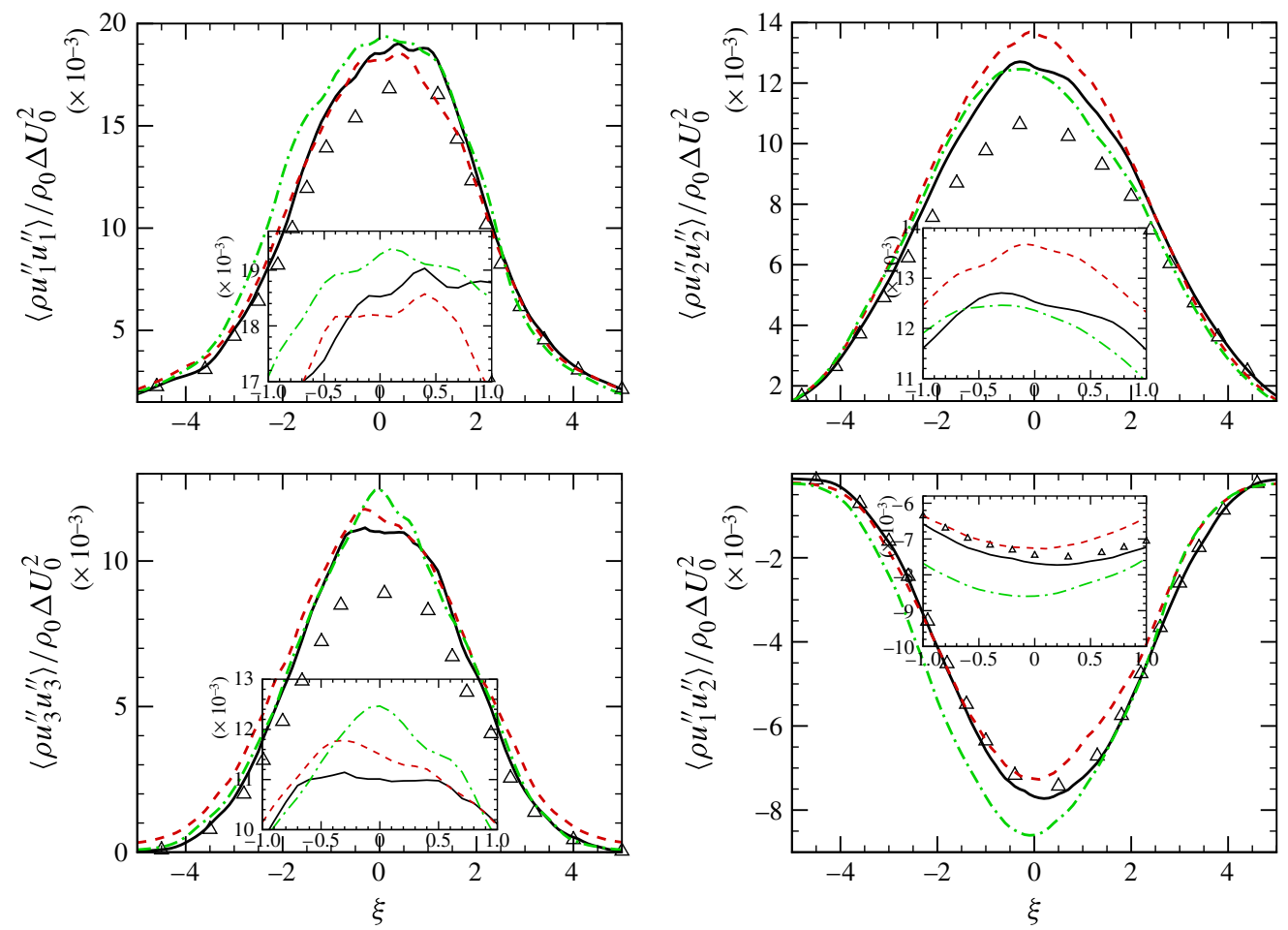

FIGURE 16. (Colour online) Reynolds stresses. - DNS; $\triangle$, filtered DNS; - - - , EFLES-NM-M8-1800; --_, EFLES-DSM-M8-1800.

spectra for these two simulations and clearly illustrates the build up of energy for EFLES-NM-F8-1800 near the filter cutoff, unlike the situation for EFLES-DSM-F81800. This indicates that the exclusive use of explicit filtering removes energy from the scales above the filter width but it does not provide the correct dissipation for scales below the filter width. Hence, an SGS model is necessary for the accurate representation of energy transfer between various scales and better prediction of TKE spectra. To further prove the necessity of SGS models, illustrated in figure 16 are the Reynolds stresses computed using EFLES-NM-F8-1800 and EFLES-DSM-F8-1800; clearly, substantial differences are evident between the Reynolds stresses calculated using these two models. Considering the fact that the SGS model plays a larger role as the $R e$ value increases, for flows at the much higher $R e$ values of engineering applications that are the ultimate target of LES, the SGS influence will be then even more significant than that shown in figure 16. Therefore, the concept of separation of numerical and modelling errors through explicit filtering is valid for these fully turbulent flows.

To summarize, grid-spacing- and discretization-order-independent results are also obtained using the EFLES approach at higher Reynolds number for the same $\bar{\Delta} / \Delta_{L E S}$ value as for $R e_{0}=600$ and in doing so, EFLES does indeed achieve its goal of separating numerical errors from SGS modelling errors. SGS models are indeed needed to correctly represent the energy transfer between various scales in the EFLES formulation. 

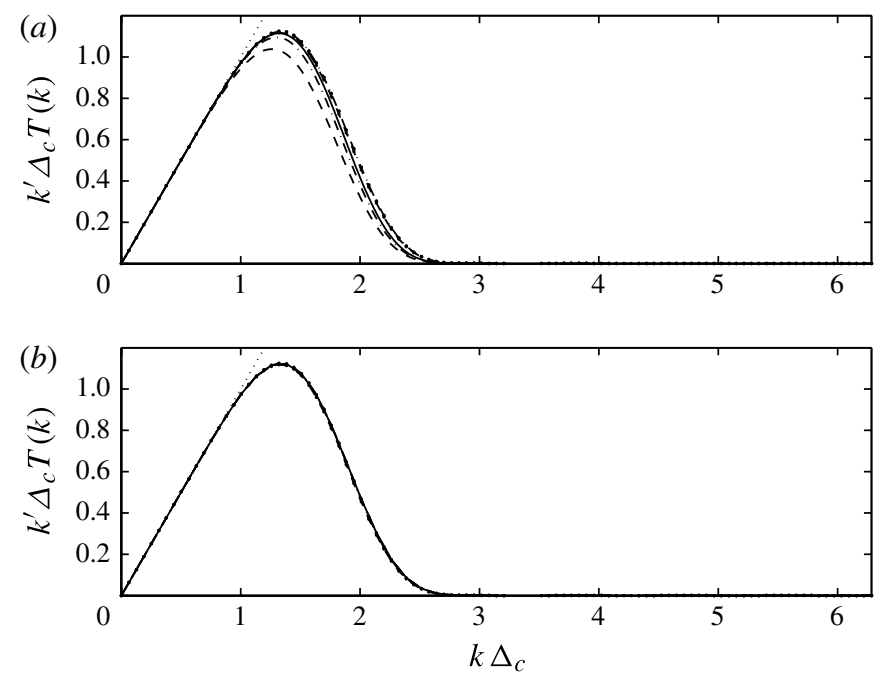

FIGURE 17. Effective modified number versus wavenumber non-dimenisionalized by $\Delta_{c}$ (coarse mesh grid spacing). (a) ---, C4; -- , C6; - , C8; ---, M4; - . -, M6; …., M8. (b) ---, F4; ---, F6; - , F8;---, M4; - . - , M6; . . . , M8.

\subsection{Analysis of reasons for EFLES performance}

We wish now to inquire: (a) Under what conditions can one obtain a grid-spacing- and discretization-order-independent solution using EFLES for separating numerical and modelling errors? (b) Can some SGS models be identified in EFLES as having more fidelity than others in terms of reproducing a known template?

\subsubsection{Under what conditions can EFLES lead to grid-spacing- and discretization-order- independent solutions for separating numerical and modelling errors?}

It has long been known that high-order schemes are required to solve the wide range of spatial and temporal scales occurring in transitional and fully turbulent flows. By its very nature, a higher-order spatial discretization scheme is a more accurate representation of a function or a derivative than a lower-order discretization scheme. For the eighth-order spatial discretization, the agreement of the results obtained with the $\mathrm{M}$ - and $\mathrm{C}$-grids means that in fact the $\mathrm{C}$-grid is sufficient to compute the EFLES solution at this discretization order and no additional accuracy is obtained from using a finer grid. For the sixth-order and the fourth-order spatial discretizations, the agreement of the results obtained with the $\mathrm{M}$ - and F-grids implies that the M-grid is necessary to compute the EFLES solution at these lower discretization orders. These results further imply that a filter-width to grid-spacing ratio of four is needed for the sixth- and fourth-order discretization schemes whereas a filter-width to grid-spacing ratio of two is sufficient for the eighth-order scheme. In figure 17, we display the product of the transfer function of the applied explicit filter and the modified wavenumber of the discretization scheme as a function of the wavenumber non-dimensionalized by the grid spacing on the C-mesh, $\Delta_{c}$. This product, which we call effective modified wavenumber, coincides in figure $17(a)$ on the $\mathrm{M}$ mesh for all discretization orders whereas the effective modified wavenumber of $\mathrm{C} 6$ and C4 does not coincide with them. In figure $17(b)$, the illustrated effective modified wavenumbers for the $\mathrm{M}$ - and $\mathrm{F}$-meshes with various discretization-order schemes 
coincide. This analysis shows that given an SGS model, as long as the effective modified wavenumber is the same for two simulations, the solutions obtained in these two simulations will be the same and be independent of the grid spacing or discretization order used in these simulations.

\subsubsection{Can some SGS models be identified as having more fidelity than others?}

Having shown that a separation of numerical and modelling errors is possible in EFLES, the question we raise is whether it is possible to identify some SGS models as having more fidelity than others. Since we found in $\S 6.4$ through examination of the objectives defined in $\S 6.3$ that EFLES-DSM and EFLES-DMM models are comparable in accuracy, we examine other quantities (i.e. $\tau_{i j}$ computed for LES and EFLES according to the definitions of either (3.5) or (3.11), depending on the formulation used) including some higher-order quantities (i.e. the TKE viscous dissipation and the TKE SGS dissipation) to inquire whether differences can be identified in these that would distinguish one of the SGS models as being more accurate than the other. As a comparison, we also consider the corresponding results for the LES-DSM and the LES-DMM to inquire whether the indications from conventional LES are different from those of EFLES.

The TKE viscous dissipation and the TKE SGS dissipation are defined as

$$
\begin{gathered}
\varepsilon^{v}=\frac{1}{2}\left(\varepsilon_{11}+\varepsilon_{22}+\varepsilon_{33}\right) \quad \text { where } \varepsilon_{i j}=\left\langle\sigma_{j k}^{\prime} \frac{\partial u_{i}^{\prime \prime}}{\partial x_{k}}+\sigma_{i k}^{\prime} \frac{\partial u_{j}^{\prime \prime}}{\partial x_{k}}\right\rangle \\
\varepsilon^{S G S}=\frac{1}{2}\left(\varepsilon_{11}^{S G S}+\varepsilon_{22}^{S G S}+\varepsilon_{33}^{S G S}\right) \quad \text { where } \varepsilon_{i j}^{S G S}=\left\langle\tau_{j k}^{\prime} \frac{\partial u_{i}^{\prime \prime}}{\partial x_{k}}+\tau_{i k}^{\prime} \frac{\partial u_{j}^{\prime \prime}}{\partial x_{k}}\right\rangle .
\end{gathered}
$$

Consideration of figure 18 for the LES-DSM shows that the C-grid computations bunch together, as also do, separately, the M-grid computations, and that the M-grid computations have better agreement with the template as the C-grid results severely underpredict $\tau_{12}$. For LES-DMM, there is also a bunching of C-grid or $\mathrm{M}$-grid computations, respectively; the C-grid predictions severely overestimate $\tau_{11}$ and $\left|\tau_{12}\right|$, while the M-grid calculations moderately overestimate these values. Based on these results, it would appear that the SGS model evaluation indicates that the LES-DSM model is overall more accurate, at least for the M-grid.

In contrast to conventional LES, figure 19, shows that for EFLES-DSM all C-grid and M-grid computations bunch together, with differences between M-grid and some $\mathrm{C}$-grid calculations observable only in the central layer region. This finding is in concert with the EFLES spectra illustrated in figure 9. Regarding model performance, for the EFLES-DSM model, with the exception of C4, C6 and to a much smaller extent C8, all EFLES predictions agree for $\tau_{11}$, though it is overpredicted. The same coincidence of all EFLES simulations as for $\tau_{11}$ (i.e. excluding C4, C6 and to a much smaller degree C8) is observed for $\tau_{12}$, but the EFLES predictions overestimate by as much as a factor of two the FDNS. Similarly, all stresses predicted by the EFLES-DMM model agree, except for the C4, C6 and to a significantly lesser extent C8. Thus, even for $\tau_{i j}$, the EFLES-DMM model performance is similar to that of the EFLES-DSM model, giving a different indication from that provided by conventional LES.

Figure 20 illustrates $\varepsilon^{S G S}$ and $\varepsilon^{v}$ for LES-DSM which clearly overpredicts the same quantities as computed from FDNS by a factor of 1.5 on the F-mesh and by as much as $70 \%$ on the C-mesh. In contrast, figure 21 illustrates $\varepsilon^{v}$ and $\varepsilon^{S G S}$ of various models in the EFLES cases. For EFLES-DSM, both $\varepsilon^{S G S}$ and $\varepsilon^{v}$ coincide for all cases except for $\mathrm{C} 4$ and $\mathrm{C} 6$ which show small differences from the 
(a)

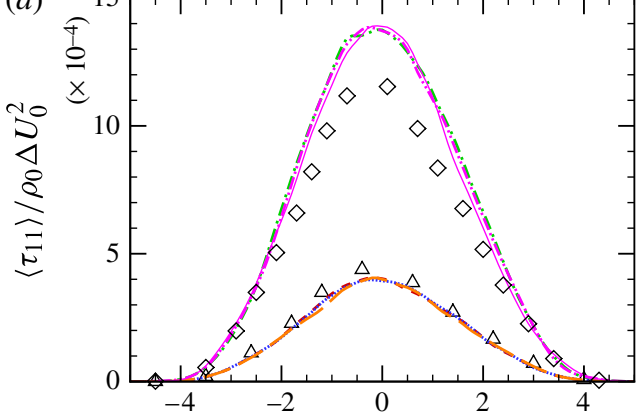

(c)

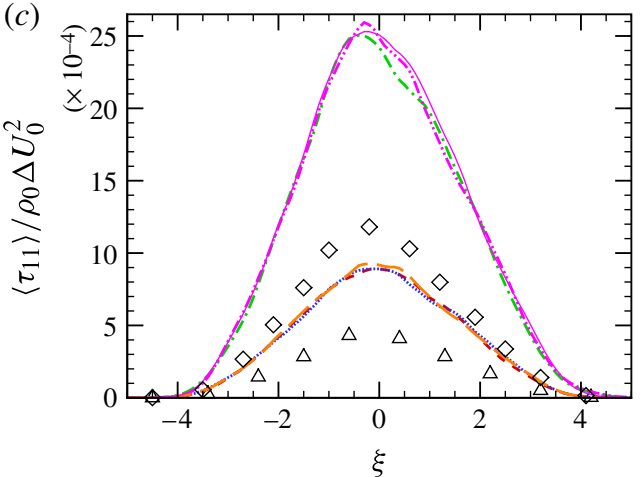

(b)
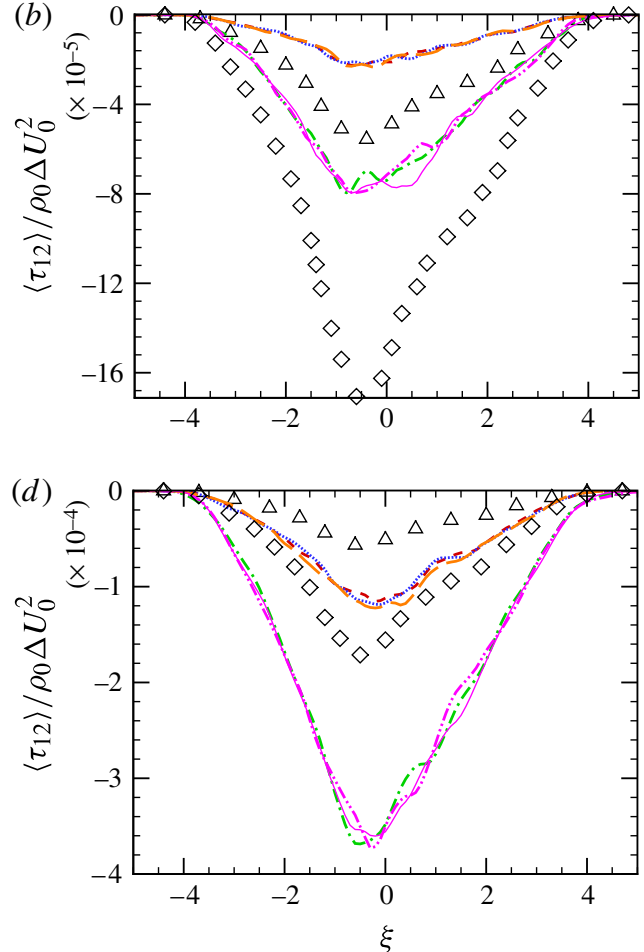

FIGURE 18. (Colour online) SGS stresses. $\triangle$, filtered DNS with $\bar{\Delta}=2 \Delta_{D N S} ; \diamond$, filtered DNS with $\bar{\Delta}=4 \Delta_{D N S} .(a, b) \ldots$ DSM-M6-600; -..., LES-DSM-C6-600; _ _ , LES-DSM-M4-600; — , LES-DSMC4-600. $(c, d)$ - - - , LES-DMM-M8-600; - - , LES-DMM-C8-600; ........., LES-DMMM6-600; -..-, LES-DMM-C6-600; _ _ , LES-DMM-M4-600; _ , LES-DMM-C4600 .

grid-converged results. For the EFLES-DMM model, the difference in $\varepsilon^{S G S}$ prediction for C4 and C6 from other cases is larger, which perhaps explains the observed larger differences in the Reynolds stress prediction on the C-mesh as compared to the ones by EFLES-DSM. The EFLES-DMM model predicts slightly larger $\varepsilon^{S G S}$ compared to the EFLES-DSM model, which probably accounts for the small difference in the grid independent Reynolds stress values obtained using these models. Globally, neither $\varepsilon^{S G S}$ nor $\varepsilon^{v}$ allows a discrimination between EFLES-DSM and EFLES-DMM model. Therefore, the evidence presented here supports the view that for EFLES, the SGS model is inconsequential provided it introduces the correct dissipation into EFLES. Such a conclusion could not be obtained from conventional LES since modelling and numerical errors are coupled.

\section{Summary and conclusions}

A large-eddy simulation (LES) formulation has been developed with the goal of obtaining grid-spacing-independent results from such computations; the idea is that these results should only depend on the filter width which specifies the smallest scale at which resolved information is desired. Unlike in conventional LES, the small-scaleproducing nonlinear terms in the governing equations are explicitly filtered, and this 
(a)
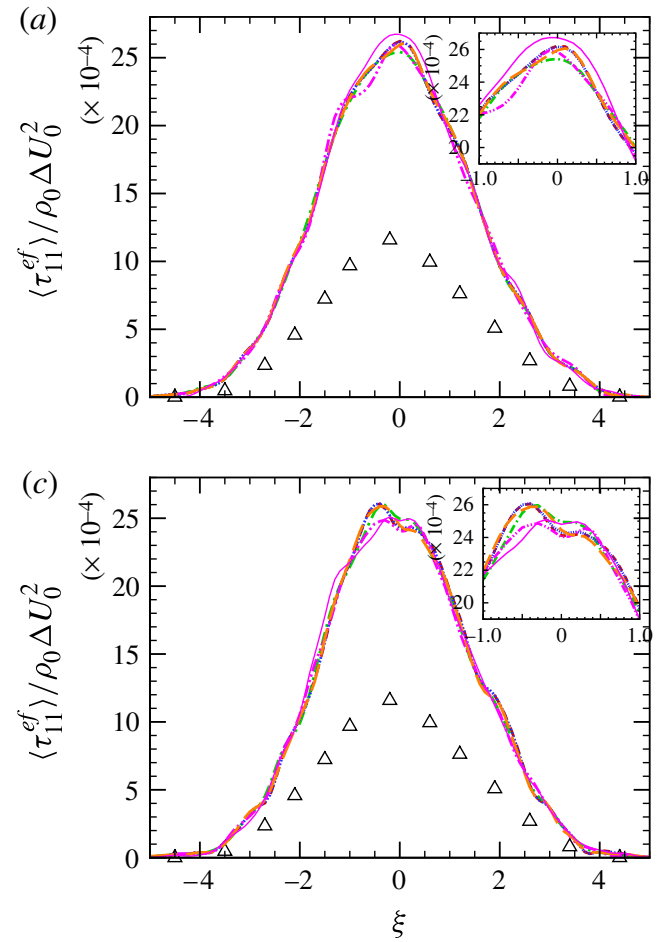
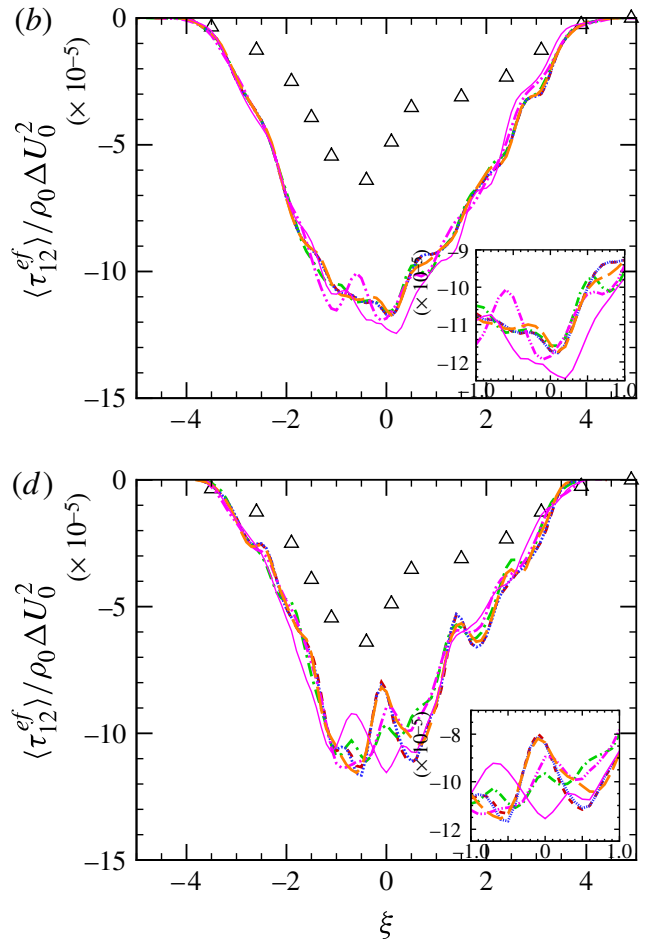

FIGURE 19. (Colour online) SGS stresses. $\triangle$, filtered DNS. $(a, b)---$, EFLES-DSM-M8$600 ;---$, EFLES-DSM-C8-600; , EFLES-DSM-M6-600; -..-, EFLES-DSM-C6$600 ;-1-$, EFLES-DSM-M4-600; — EFLES-DSM-C4-600. $(c, d)$ - - - , EFLESDMM-M8-600; ---, EFLES-DMM-C8-600; ........, EFLES-DMM-M6-600; -..-, EFLESDMM-C6-600; — _ , EFLES-DMM-M4-600; , EFLES-DMM-C4-600.

procedure is applied both to the differential equations and the equation of state; we call such formulation EFLES. To examine the properties of such computations, a template database was first created from direct numerical simulation (DNS) portraying the evolution of an initially-perturbed three-dimensional shear layer to a state of self-similar growth; this template has served as the basis for comparison determining the accuracy of both conventional LES and EFLES.

Both conventional LES and EFLES computations have been conducted with several SGS models. Because the definition of the SGS terms is different in EFLES from the conventional LES, only some of the typically used SGS models could be used in EFLES computations. For conventional LES, the filter width was chosen to be equal to the grid spacing. For EFLES, the filter width was independent of the grid spacing. Results were obtained at three different spatial-order discretizations - fourth-order, sixth-order and eighth-order - and with either a coarse or a medium grid for conventional LES, and with a coarse, medium or a fine grid for EFLES. Examination of these results revealed the following. (a) Unlike for conventional LES where the results are always grid-spacing dependent, the EFLES results are gridspacing independent for the eighth-order spatial discretization on all three meshes, while they are grid-spacing independent on the medium and fine meshes for the fourth- and sixth-order spatial discretizations. (b) LES and EFLES provide different conclusions in terms of evaluating SGS models' accuracy with respect to a template. 
(a)

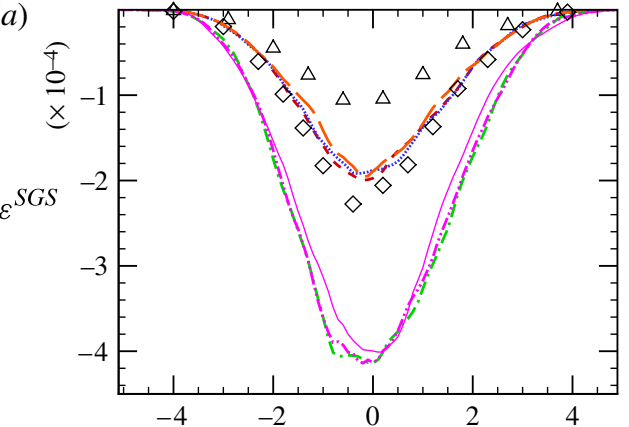

(c)

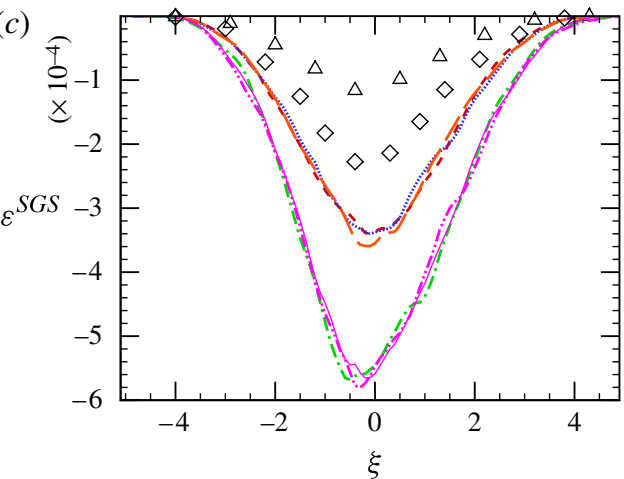

(b)

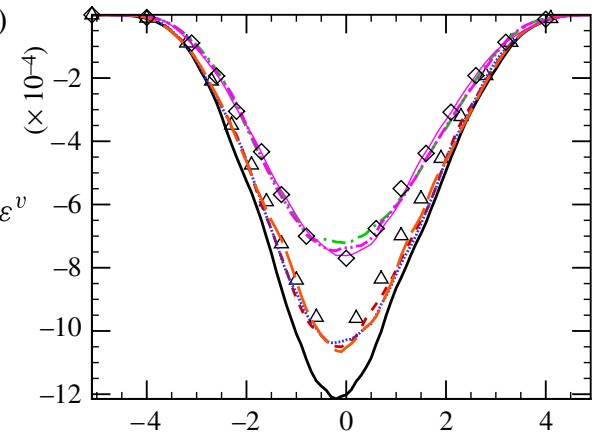

(d)

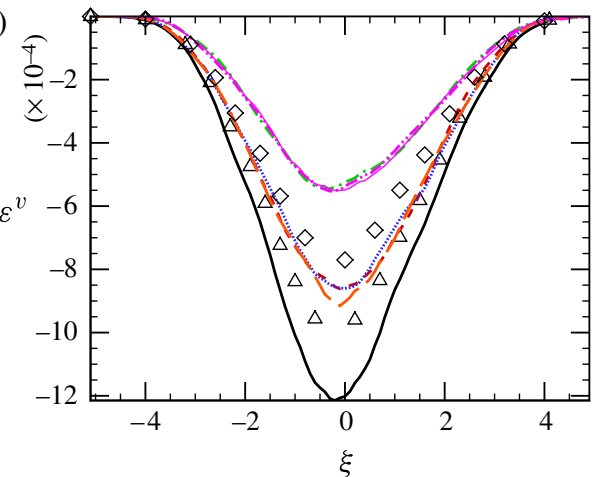

FIGURE 20. (Colour online) Viscous and SGS dissipation. - DNS; $\triangle$, filtered DNS with $\bar{\Delta}=2 \Delta_{D N S} ; \diamond$, filtered DNS with $\bar{\Delta}=4 \Delta_{D N S} \cdot(a, b)$ - $~-$, , LES-DSM-M8-600; --- , LESDSM-C8-600; ........., LES-DSM-M6-600; -..., LES-DSM-C6-600; — - , LES-DSMM4-600; — , LES-DSM-C4-600. $(c, d)$ _ - - , LES-DMM-M8-600; - - - , LES-DMMC8-600; ........., LES-DMM-M6-600; -..., LES-DMM-C6-600; — - , LES-DMM-M4600 , LES-DMM-C4-600.

For conventional LES this evaluation is the result of both SGS modelling and numerical errors, whereas for EFLES, this is the result of SGS modelling capabilities. (c) Unlike for conventional LES where the results are always discretization-order dependent, the EFLES predictions are discretization-order independent for the medium and the fine grids employed. For EFLES, we found that the filter-width to gridspacing ratio needed for obtaining the grid-spacing-independent solution is a function of the discretization order used. A filter-width to grid-spacing ratio of four is needed to obtain grid-spacing-independent results when using the fourth- or sixth-order discretization scheme; however, a filter-width to grid-spacing ratio of two is sufficient to obtain grid-spacing-independent results when using the eighth-order discretization scheme. These findings have been explained by analysing the modified wavenumber of the discretization and the transfer function of the applied filter.

Since EFLES is computationally more intensive than conventional LES (Gullbrand \& Chow 2003; Lund 2003), it is the user who must decide whether the additional expense warrants the ability for SGS model evaluation.

\section{Acknowledgements}

This work was conducted at the Jet Propulsion Laboratory, California Institute of Technology and sponsored by the National Aeronautics and Space 
(a)

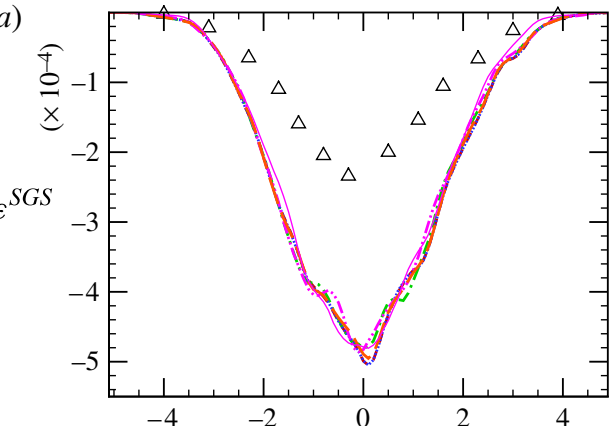

(c)

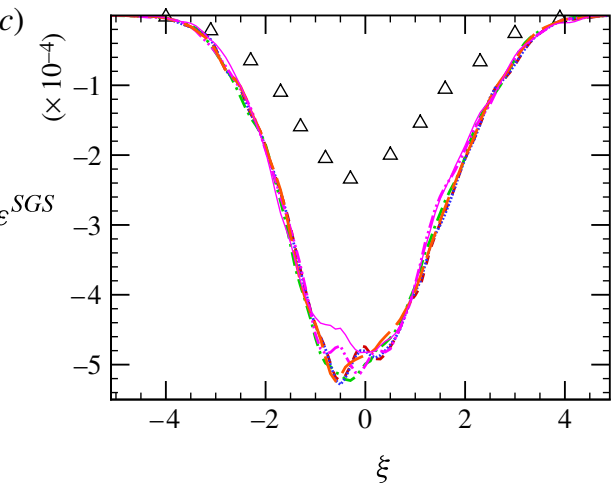

(b)

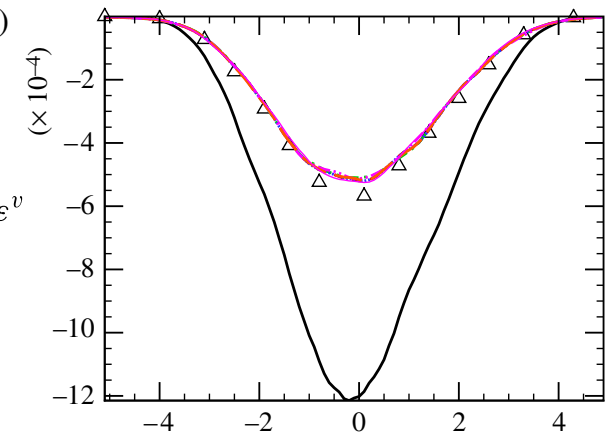

(d)

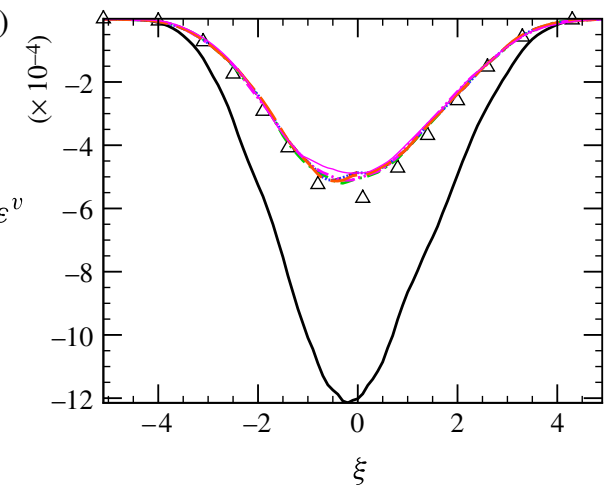

FIGURE 21. (Colour online) Viscous and SGS dissipation. - DNS; $\triangle$, filtered DNS. $(a, b)$ - - - , EFLES-DSM-M8-600; -- , EFLES-DSM-C8-600; ........, EFLES-DSM-M6$600 ;-\cdots-$, EFLES-DSM-C6-600; —_ , EFLES-DSM-M4-600; — , EFLES-DSMC4-600. $(c, d)$ - - - , EFLES-DMM-M8-600; - - -, EFLES-DMM-C8-600; ........., EFLESDMM-M6-600; -..., EFLES-DMM-C6-600; _ — , EFLES-DMM-M4-600; EFLES-DMM-C4-600.

Administration (NASA) under the Fundamental Aeronautics Program, Subsonic Wing Program from NASA Glenn Research Center with Drs Dan Bulzan and NanSuey Liu serving as program monitors and by the NASA Exploration Systems Mission Directorate/Advanced Capabilities Division under the LASER program. The computational resources were provided by the JPL Supercomputing Center and by the NASA AMES Supercomputing Center.

\section{Appendix. Explicit filter details}

We list the coefficients of the constructed eighth-order filter, for various filter widths below. For the filter applied on the coarse mesh, the weights are

$$
\begin{gathered}
a_{0}=\frac{7.68359375}{14}, \quad a_{1}=\frac{4.1015625}{14}, \quad a_{2}=\frac{-0.5126953125}{14}, \quad a_{3}=\frac{-0.68359375}{14}, \\
a_{4}=\frac{0.205078125}{14}, \quad a_{5}=\frac{0.08203125}{14} \text { and } a_{6}=\frac{-0.034179687}{14} .
\end{gathered}
$$

The weights for the filter applied on the medium mesh are

$$
a_{0}=\frac{7.68359375}{28}, \quad a_{1}=\frac{6.648157262171023}{28}, \quad a_{2}=\frac{4.1015625}{28},
$$




$$
\begin{gathered}
a_{3}=\frac{1.329606760615415}{28}, \quad a_{4}=\frac{-0.5126953125}{28}, \quad a_{5}=\frac{-1.048009348513550}{28}, \\
a_{6}=\frac{-0.68359375}{28}, \quad a_{7}=\frac{-0.112434387329164}{28}, \quad a_{8}=\frac{0.205078125}{28}, \\
a_{9}=\frac{0.210987824202490}{28}, \quad a_{10}=\frac{0.08203125}{28}, \quad a_{11}=\frac{-0.015146223534823}{28}, \\
a_{12}=\frac{-0.034179687}{28} \quad \text { and } \quad a_{13}=\frac{-0.013161887611393}{28} .
\end{gathered}
$$

The weights for the filter applied on the fine mesh are

$$
\begin{aligned}
& a_{0}=\frac{7.68359375}{56}, \quad a_{1}=\frac{7.415102647441697}{56}, \quad a_{2}=\frac{6.648157262171023}{56}, \\
& a_{3}=\frac{5.491045690680181}{56}, \quad a_{4}=\frac{4.1015625}{56}, \quad a_{5}=\frac{2.658285586376428}{56}, \\
& a_{6}=\frac{1.329606760615415}{56}, \quad a_{7}=\frac{0.246950010045}{56}, \quad a_{8}=\frac{-0.5126953125}{56}, \\
& a_{9}=\frac{-0.932223001516977}{56}, \quad a_{10}=\frac{-1.048009348513550}{56} \text {, } \\
& a_{11}=\frac{-0.934412015092289}{56}, \quad a_{12}=\frac{-0.68359375}{56}, \\
& a_{13}=\frac{-0.385545944401394}{56}, \quad a_{14}=\frac{-0.112434387329164}{56}, \\
& a_{15}=\frac{0.090231885502304}{56}, \quad a_{16}=\frac{0.205078125}{56}, \quad a_{17}=\frac{0.238232035865567}{56} \text {, } \\
& a_{18}=\frac{0.210987824202490}{56}, \quad a_{19}=\frac{0.150524243276105}{56}, \quad a_{20}=\frac{0.08203125}{56}, \\
& a_{21}=\frac{0.023687502284170}{56}, \quad a_{22}=\frac{-0.015146223534823}{56}, \\
& a_{23}=\frac{-0.033066577695188}{56}, \quad a_{24}=\frac{-0.034179687}{56}, \quad a_{25}=\frac{-0.025226999642401}{56}, \\
& a_{26}=\frac{-0.013161887611393}{56} \text { and } a_{27}=\frac{-0.003585063123203}{56} \text {. }
\end{aligned}
$$

REFERENCES

BARdina, J., FERZIGER, J. \& REYnolds, W. 1980 Improved subgrid scale models for large eddy simulation. AIAA Paper 80-1357.

Bose, S. T., Moin, P. \& You, D. 2010 Grid-independent large-eddy simulation using explicit filtering. Phys. Fluids 22, 105103-1-11.

Carati, D, Winckelmans, G. S. \& Jeanmart, H. 2001 On the modelling of the subgrid-scale and filtered-scale stress tensors in large-eddy simulation. J. Fluid Mech. 442, 119-138.

Chow, F. K. \& MoIn, P. 2003 A further study of numerical errors in large-eddy simulations. J. Comput. Phys. 184, 366-380.

Clark, R., Ferziger, J. \& Reynolds, W. 1979 Evaluation of subgrid-scale models using an accurately simulated turbulent flow. J. Fluid Mech. 91 (1), 1-16.

Fox, R. O. 2003 Computational Models for Turbulent Reactive Flows. Cambridge University Press.

Germano, M., Piomelli, U., Moin, P. \& Cabot, W. 1991 A dynamic subgrid-scale eddy viscosity model. Phys. Fluids A 3 (7), 1760-1765. 
Geurts, B. J. 1997 Inverse modelling for large-eddy simulation. Phys. Fluids 9 (12), 3585-3587.

Geurts, B. J. \& Frohlich, J 2002 A framework for predicting accuracy limitations in large-eddy simulation. Phys. Fluids 14 (6), L41-L44.

GhosAl, S. 1996 An analysis of numerical errors in large-eddy simulations of turbulence. J. Comput. Phys. 125, 187-206.

GullbRAnd, J. \& CHOW, F. K. 2003 The effect of numerical errors and turbulence models in large-eddy simulations of channel flow, with and without explicit filtering. J. Fluid Mech. 495, 323-341.

Kennedy, C. \& CARpenter, M. 1994 Several new numerical methods for compressible shear layer simulations. Appl. Numer. Maths 14, 397-433.

Kennedy, C. A. \& GRUber, A. 2008 Reducing aliasing formulations of the convective terms within the Navier-Stokes equations for a compressible fluid. J. Comput. Phys. 227, $1676-1700$.

IsRaeli, M. \& Orszag, S. A. 1981 Approximation of radiation boundary conditions. J. Comput. Phys. 41, 115-135.

LESIEUR, M. 1997 Turbulence in Fluids. Kluwer.

LILLY, D. 1992 A proposed modification of the Germano subgrid-scale closure method. Phys. Fluids A 4 (3), 633-635.

LiU, S., Meneveau, C. \& Katz, J. 1994 On the properties of similarity subgrid-scale models as deduced from measurements in a turbulent jet. J. Fluid Mech. 275, 83-119.

LUnd, T. S. 2003 The use of explicit filters in large eddy Simulation. Comput. Maths Applics. 46, 603-616.

Magnient, J-C., Sagaut, P. \& Deville, M. 2001 A study of built-in filter for some eddy viscosity models in large-eddy simulation. Phys. Fluids 13 (5), 1440-1449.

Martin, M. P., Piomelli, U. \& Candler, G. V. 2000 Subgrid-scale models for compressible Large-Eddy Simulation. J. Theor. Comput. Fluid Dyn. 13, 361-376.

Mathew, J., Lechner, R., Foysi, H., Sesterhenn, J. \& Friedrich, R. 2003 An explicit filtering method for large eddy simulation of compressible flows. Phys. Fluids 15 (8), 2279-2289.

Mattsson, K. \& Nordstrom, J. 2004 Summation by parts operators for finite difference approximations of second derivatives. J. Comput. Phys. 199, 503-540.

Meyers, J., Geurts, B. J. \& Baelmans, M. 2003 Database analysis of errors in large-eddy simulation. Phys. Fluids 15 (9), 2740-2755.

Meyers, J., Geurts, B. J. \& Baelmans, M. 2005 Optimality of the dynamic procedure for large-eddy simulations. Phys. Fluids 17, 0451108-1-9.

Meyers, J., Sagaut, P. \& Geurts, B. J. 2006 Optimal model parameters for multi-objective Large-Eddy Simulations. Phys. Fluids 18, 095103-1-12.

OKONG'O, N. \& BELlAN, J. 2004 Consistent large eddy simulation of a temporal mixing layer laden with evapourating drops. Part 1. Direct numerical simulation, formulation and a priori analysis. J. Fluid Mech. 499, 1-47.

Pantano, C. \& SARKar, S. 2002 A Study of Compressibility Effects in the High-Speed, Turbulent Shear Layer Using Direct Simulation. J. Fluid Mech. 451, 329-371.

Poinsot, T. \& LELE, S. 1992 Boundary conditions for direct simulations of compressible viscous flows. J. Comput. Phys. 101, 104-129.

Pope, S. B. 2000 Turbulent Flows. Cambridge University Press.

Pope, S. B. 2004 Ten questions concerning the large-eddy simulation of turbulent flows. New J. Phys. 6, 35-59.

Pope, S. B. 2010 Self-conditioned fields for large-eddy simulations of turbulent flows. J. Fluid Mech. 652, 139-169.

Pruett, C., Sochacki, J. \& Adams, N. 2001 On Taylor-series expansions of residual stress. Phys. Fluids 13 (9), 2578-2589.

Sagaut, P \& DeCK, S. 2009 Large eddy simulation for aerodynamics: status and perspectives. Phil. Trans. R. Soc. Lond. A367, 2849-2860.

SMAGORINKSY, J. 1963 General circulation experiments with the primitive equations. Part 1, basic experiments. Mon. Weath. Rev. 91, 99-164. 
SMAGORINKSY, J. 1993 Some historical remarks on the use of nonlinear viscosities. In Large Eddy Simulation of Complex Engineering and Geophysical Flows, Chap. 1 (ed. B. Galperin \& S. Orszag), pp. 3-36. Cambridge University Press.

Stolz, S. \& AdAms, N. A. 1999 An approximate deconvolution procedure for large-eddy simulation. Phys. Fluids 11 (7), 1699-1701.

Stolz, S., AdAms, N. A. \& Kleiser, L. 2001 An approximate deconvolution model for large-eddy simulation with application to incompressible wall-bounded flows. Phys. Fluids $\mathbf{1 3}$ (4), 997-1015.

Vanella, M., Piomelli, U. \& Balaras, E. 2008 Effect of grid discontinuities on large-eddy simulation statistics and flow fields. J. Turbul. 9 (32), 1-23.

Vreman, B., Geurts, B. \& Kuerten, H. 1996 Comparison of numerical schemes in large-eddy simulation of the temporal mixing layer. Intl J. Numer. Meth. Fluids 22, 297-311.

Winckelmans, G. S., Wray, A. A, Vasilev, O. V. \& Jeanmart, H. 2001 Explicit-filtering large-eddy simulation using the tensor-diffusivity model supplemented by a dynamic Smagorinsky term. Phys. Fluids 13 (5), 1385-1403.

Yoshizawa, A. 1986 Statistical theory for compressible turbulent shear flows, with the application to subgrid modelling. Phys. Fluids 29 (7), 2152-2164. 